CBP Tech Note 197, LCC-0032

November 1999.

\title{
PEP-II RF Cavity Revisited
}

\author{
R.A. Rimmer, G. Koehler, D. Li, N. Hartman, \\ LBNL \\ N. Folwell , J. Hodgson, K. Ko, B. McCandless, \\ SLAC
}

Abstract

This report describes the results of numerical simulations of the PEP-II RF cavity performed after the completion of the construction phase of the project and comparisons are made to previous calculations and measured results. These analyses were performed to evaluate new calculation techniques for the HOM distribution and RF surface heating that were not available at the time of the original design. These include the use of a high frequency electromagnetic element in ANSYS and the new Omega 3P code to study wall losses, and the development of broadband time domain simulation methods in MAFIA for the HOM loading. The computed HOM spectrum is compared with cavity measurements and observed beam-induced signals. The cavity fabrication method is reviewed, with the benefit of hindsight, and simplifications are discussed.

Introduction

The PEP-II RF cavity is among the first designs with strong damping of the higher order modes (HOMs), which are now seeing operation in the high-current $\mathrm{e}+\mathrm{e}$ - factories around the world $[1,2]$. This design has already proved to be very successful in the reduction of coupled-bunch instability growth rates, contributing to the rapid commissioning of the rings and the early attainment of high luminosity. The technology developed for this project has already been put to further use for the ALS third harmonic cavity upgrade [3], and is a natural candidate for future projects such as light sources, storage ring based colliders, and linear collider damping rings [4]. Indeed a scaled version of the PEP-II design was used as the baseline model in the NLC ZDR [5]. Many lessons were learned during the design, manufacture, and commissioning of these cavities, and it is prudent to consider these when adapting the design to new applications [6]. The availability of better design tools and faster computers also now allows more detailed study of some aspects of the design. 
Calculation of RF properties and HOMs

One of the most difficult tasks in designing a strongly HOM damped RF cavity is to ensure that all longitudinal and transversely deflecting modes are adequately damped, up to and possibly above the beam pipe cutoff frequency. At the same time the efficiency of the accelerating mode must be kept as high as possible. In the PEP-II cavity design phase the coupling to the cavity HOMs was estimated one mode at a time by a perturbation method [7] requiring multiple simulation results per mode, which was very time consuming and limited the analysis to only the lower frequency modes. In fact, not all the modes below cutoff could be calculated this way. The design was verified for production by extensive measurements of a full-size cold test model [8], which confirmed the calculated results and showed that the damping scheme was successful for all the harmful modes below cutoff.

With advances in computer speed and new calculation techniques it is now possible to look at the broad-band impedance spectrum of the damped cavity in a single run and estimate the strength of all the modes, so long as they do not have very high Q's [9]. This allows the investigation of geometry changes and some degree of optimization to be performed on a reasonable time scale. The method involves a simulation in the time domain wherein the structure is excited by a short bunch passing through it, either on axis (for the longitudinal modes) or off axis (for the deflecting modes), and the long range wakefield is computed and recorded for many meters behind the bunch. This time record contains the amplitude of excitation and exponential decay of all of the modes and a Fourier transform of this signal, normalized to the bunch spectrum, reveals the broad-band impedance spectrum of the cavity. The frequency resolution is determined by the length of the wakefield recorded, and is limited only by computer time and, eventually, by the onset of numerical instabilities in the simulation. Any mode still ringing with appreciable amplitude at the end of the recorded time will be truncated and artificially broadened in the spectrum, so care must be taken in interpreting the results. In order to check the validity of these tools the PEP-II cavity geometry was modeled and the results compared with the known cavity properties.

Figure 1 shows the calculated longitudinal impedance spectrum using this method for the PEP-II geometry, including coupler, compared with the values estimated from the measurements of the cold-test model. Note that all of the modes seem to be well resolved (except the fundamental mode which of course has a very high Q). 


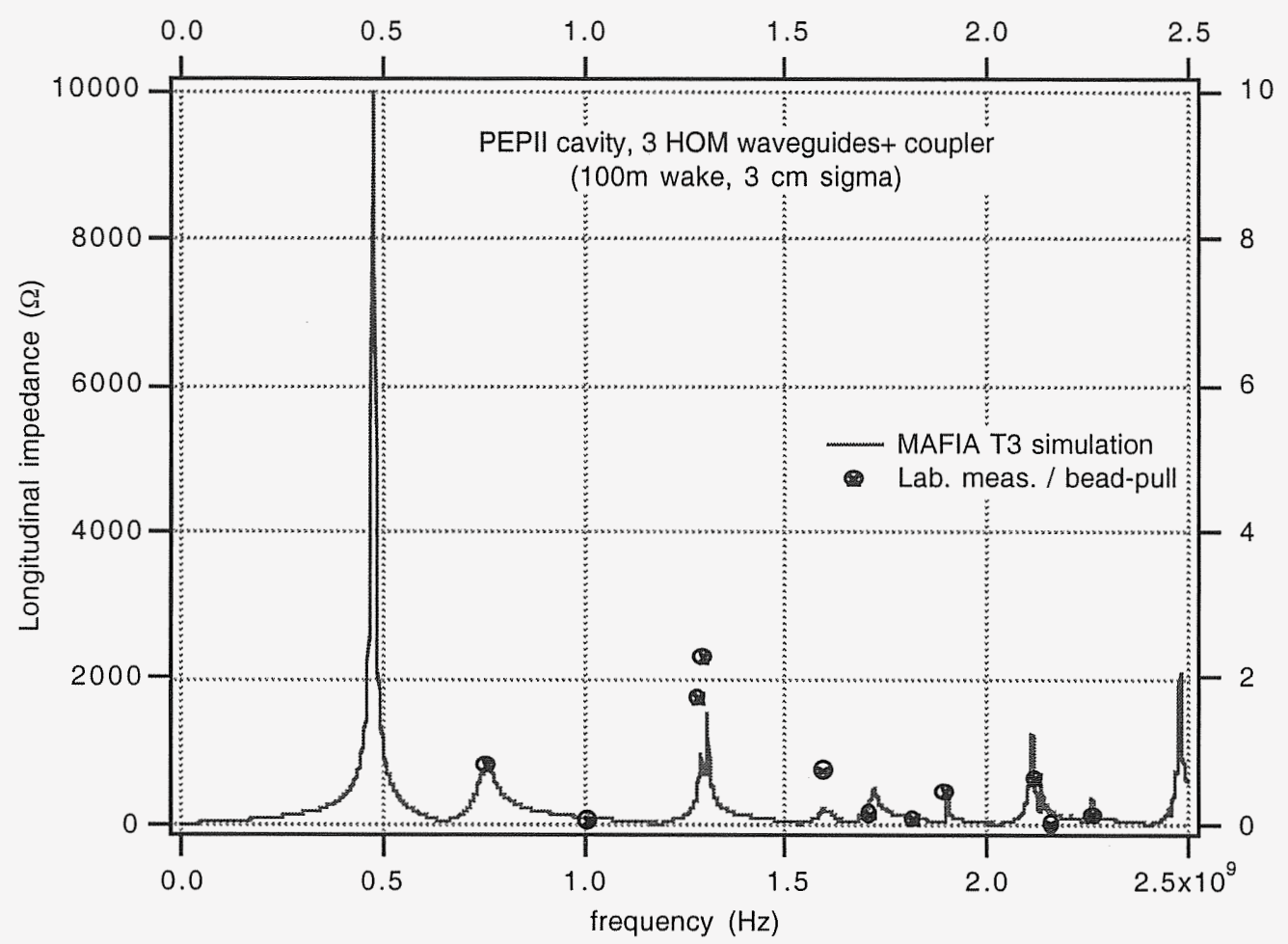

Figure 1. Calculated longitudinal impedance spectrum plus worst case estimates of modes measured in the first PEP-II cavity.

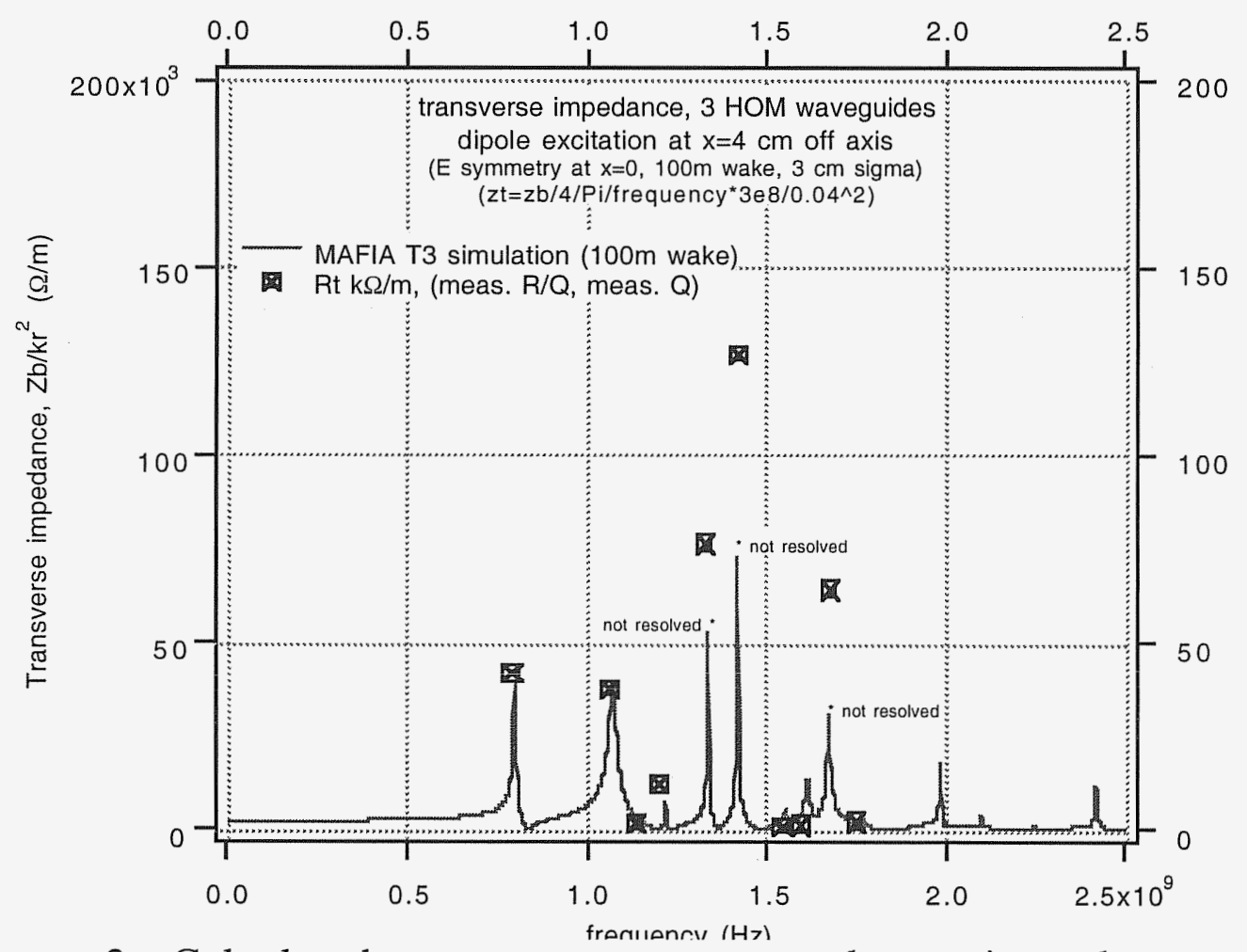

Figure 2. Calculated transverse spectrum plus estimated transverse impedance of modes measured in the first PEP-II cavity. 
The measurements generally agree well with the calculated spectrum, and where there is disagreement it may be because the measured results, such as the $\mathrm{Q}$ values extracted from the recorded spectra, were generally erred on the conservative side to get a worst case estimate. A simple check to see if the peaks are resolved is to run the simulation for a longer time. If the peaks stay the same they may be trusted but if the amplitude changes in proportion to the wake length then they are not resolved and their true amplitudes may be significantly higher. Typical simulations run on this geometry used wakes of up to $100 \mathrm{~m}$, which could be calculated overnight and resolve modes with peak widths as low as about $6 \mathrm{MHz}$, corresponding to loaded Q's from a few hundred at low frequency to about a thousand at high frequency.

Figure 2 shows the spectrum for the transverse modes calculated with a bunch off axis and a symmetry plane in the center of the cavity so that a pure dipole excitation results. (note that the excitation current is effectively double because of the "image bunch" implied by the symmetry plane and this is accounted for in the normalization of the results). In this case the lowest modes are adequately resolved with a $100 \mathrm{~m}$ wake length but some of the higher modes are not, particularly the $1335 \mathrm{MHz}, 1417 \mathrm{MHz}$ and $1672 \mathrm{MHz}$ modes. These peaks are lower than the measured values, however it should be noted that the amplitude of the peak can still be useful in the simulation as a rough indicator, for example if the waveguide geometry is changed and the peak goes down or up for the same wake length then the mode can be assumed to be more or less damped. In such cases the true amplitude of the peak can be estimated if the R/Q of the unperturbed mode is known and can be assumed to not change much with the addition of the damping ports. Alternatively the mode strength can be determined by doing a separate simulation in which the mode is excited by a constant amplitude current or voltage source in the cavity, rather than a bunch, and the quasi-linear growth of the mode amplitude can be recorded, along with the field amplitude at certain times. Once the mode pattern has been well established it is possible to compare the stored energy in the mode to the time-averaged power flow out of the HOM ports and thus estimate the external Q. This method has the advantage that the field distribution of the mode is recorded so that the shunt impedance and Qo can also be calculated. Table 1 shows a summary of the measured and calculated values for the longitudinal modes. Table 2 shows the same for the transverse modes, where both the measured and calculated impedances are for the orientation of the dipole modes which are not damped by the power coupler. 
Table 1. Impedance of longitudinal modes estimated from calculated and measured R/Q's and measured (fitted) Q's.

\begin{tabular}{|c|c|c|c|c|c|c|}
\hline Mode $\ddagger$ & $\begin{array}{l}\text { f meas. } \\
(\mathrm{MHz})\end{array}$ & $\begin{array}{c}\mathrm{R} / \mathrm{Q} \text { meas. } \\
(\Omega)\end{array}$ & $\begin{array}{l}\text { Q meas. } \\
\text { (fitted) }\end{array}$ & $\begin{array}{l}\text { Rs }(\Omega) \\
\text { (meas ) }\end{array}$ & $\begin{array}{c}f \text { calc } \\
\text { T3 } \\
(\mathrm{MHz})\end{array}$ & $\begin{array}{c}\text { Rs calc } \\
\text { T3 } \\
(\Omega)\end{array}$ \\
\hline $0-E-1$ & 475 & 117.3 & 32469 & $3.809 \mathrm{e} 6$ & 476 & n.r. \\
\hline $0-\mathrm{M}-1$ & 758 & 44.6 & $18 *$ & 810 & 760 & 869 \\
\hline $0-E-2$ & 1009 & 0.43 & 128 & 55 & 1011 & 110 \\
\hline n e w & 1283 & 6.70 & 259 & 1740 & 1291 & 969 \\
\hline $0-M-2$ & 1295 & 10.3 & 222 & 2290 & 1307 & 1496 \\
\hline $0-E-3$ & n.v. & n.v. & $30 *$ & n.v. & n.v. & n.v. \\
\hline $0-E-4$ & 1595 & 2.43 & 300 & 730 & 1597 & 212 \\
\hline $0-M-3$ & 1710 & 0.44 & 320 & 140 & 1721 & 476 \\
\hline $0-E-5$ & 1820 & 0.13 & $543 *$ & 70 & n.v. & n.v. \\
\hline $0-M-4$ & 1898 & 0.17 & 2588 & 440 & 1906 & 388 \\
\hline $0-E-6$ & 2121 & 1.82 & 338 & 620 & 2113 & 1255 \\
\hline $0-M-5$ & 2160 & 0.053 & $119^{*}$ & 6 & 2153 & 251 \\
\hline $0-E-7$ & 2265 & 0.064 & $1975 *$ & 130 & 2263 & 369 \\
\hline $0-E-8$ & 2344 & $\mathrm{n} . \mathrm{m}$ & $693 *$ & $\mathrm{n} . \mathrm{m}$. & $\mathrm{n} . \mathrm{v}$. & $\mathrm{n} . \mathrm{v}$. \\
\hline
\end{tabular}

$\ddagger E=$ electric field, $\mathrm{M}=$ magnetic field boundary condition at cavity center.

* Approx. fit or worst-case estimate, n.v. = mode not visible after damping, n.m. = mode not measured, n.r. = mode not resolved in time domain run.

Table 2. Transverse impedance of dipole modes estimated from calculated and measured R/Q's and measured Q's.

\begin{tabular}{|c|c|c|c|c|c|c|}
\hline Mode & $\begin{array}{l}\text { f meas. } \\
(\mathrm{MHz})\end{array}$ & $\begin{array}{c}\mathrm{R} / \mathrm{Q} @ 4 \mathrm{~cm} \\
\text { be ad-pull } \\
(\Omega)\end{array}$ & $\begin{array}{l}\text { Q meas. } \\
\text { (fitted) }\end{array}$ & $\begin{array}{l}\mathrm{R}_{\perp} \text { meas. }{ }^{\dagger} \\
(\mathrm{k} \Omega / \mathrm{m})\end{array}$ & $\begin{array}{c}\text { fcalc } \\
\text { T3 } \\
(\mathrm{MHz}) \\
\end{array}$ & $\begin{array}{c}\mathrm{R}_{\perp} \text { calc. } \\
\mathrm{T} 3 \\
(\mathrm{k} \Omega / \mathrm{m})\end{array}$ \\
\hline $1-\mathrm{M}-1$ & n.v. & $\mathrm{n} . \mathrm{v}$ & n.v. & n.v. & n.v. & n.v. \\
\hline $1-E-1$ & 792 & 9.69 & $115^{*}$ & 42.0 & 800 & 38.7 \\
\hline $1-M-2$ & 1063 & 50.4 & 27 & 38.0 & 1071 & 40.1 \\
\hline $1-E-2$ & 1133 & 1.29 & $54 *$ & 1.82 & n.v. & n.v. \\
\hline $1-M-3$ & 1202 & 0.56 & 871 & 12.2 & 1218 & 7.20 \\
\hline $1-E-3$ & 1327 & 5.58 & 611 & 76.7 & 1335 & $52.8^{\text {**** }}$ \\
\hline $1-M-4$ & 1420 & 5.31 & 1138 & 126.9 & 1417 & $72.9^{* * *}$ \\
\hline $1-E-4$ & 1542 & 0.50 & $92 *$ & 0.89 & 1553 & 5.35 \\
\hline $1-E-5$ & 1595 & 0.51 & $145^{*}$ & 1.39 & 1611 & 14.0 \\
\hline $1-E-6$ & 1676 & 4.63 & 783 & 64.5 & 1672 & $30.8^{* * *}$ \\
\hline $1-M-5$ & 1723 & n.v. & $446 *$ & n.v. & n.v. & n.v. \\
\hline $1-M-6$ & 1749 & 0.10 & 1317 & 2.31 & 1774 & 2.79 \\
\hline
\end{tabular}

$\ddagger$ orientation of mode not affected by coupler. * Approx. fit or worst-case estimate. ${ }^{\dagger} \mathrm{R}_{\perp}=\mathrm{R} /\left(\mathrm{Qkr}^{2}\right) \times \mathrm{Q}_{\mathrm{L}}$, n.v. = mode not visible after damping,

n.m. = mode not measured, ${ }^{* *}$ mode not fully resolved in time domain run. 
Operation in PEP-II

The PEP-II cavities are now in routine operation at SLAC at high current and with well behaved beam. Evidence from the longitudinal and transverse feedback systems suggest that the actual mode strengths are consistent with expectations from the cold-model measurements [10]. During the commissioning it was also possible to observe the signals reaching one of the HOM loads through a pick-up antenna close to the load material, included for that purpose. With a single bunch in the machine all the longitudinal modes are excited and the mode spectrum was clearly visible on a spectrum analyzer connected to the HOM probe. Figure 3 shows the HOM signal recorded by the spectrum analyzer and the calculated spectrum reaching the port in the MAFIA time domain simulation for frequencies below the beam pipe cutoff $(2378 \mathrm{MHz})$.

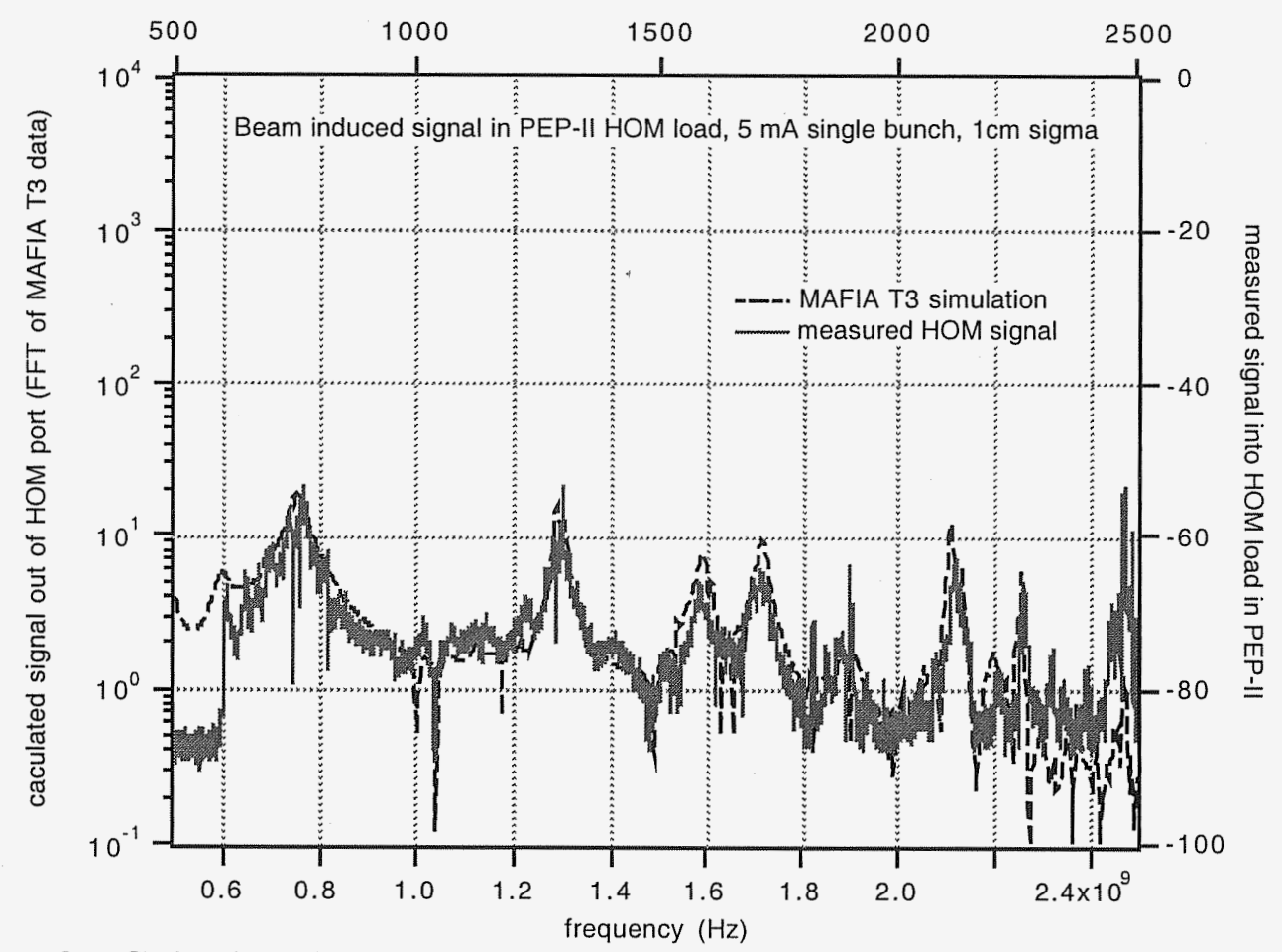

Figure 3. Calculated mode spectrum reaching the HOM load compared with signal measured with beam (modes below beam pipe cutoff).

There is very good agreement between the simulation and measurement in the distribution, structure and relative amplitudes of the peaks, although the absolute amplitude of the measured signal is uncalibrated and is adjusted on the plots to approximately match the calculated spectrum. Note that the measured signal clearly shows the cutoff of the HOM waveguide at $600 \mathrm{MHz}$; the simulated port 
boundary in the MAFIA model is closer to the cavity and therefore shows more penetration by the frequencies below cutoff. While there is good general agreement between the spectra there is additional structure in the measured data, which was also visible in the measurements of the cold-test model. This comes from coupling between the cavity modes and weak resonances in the coupling box, which is simplified in the MAFIA model and has a broad-band termination. In reality there are reflections from the window at frequencies other than the operating frequency and these cause weak resonances in the coupling box at HOM frequencies.

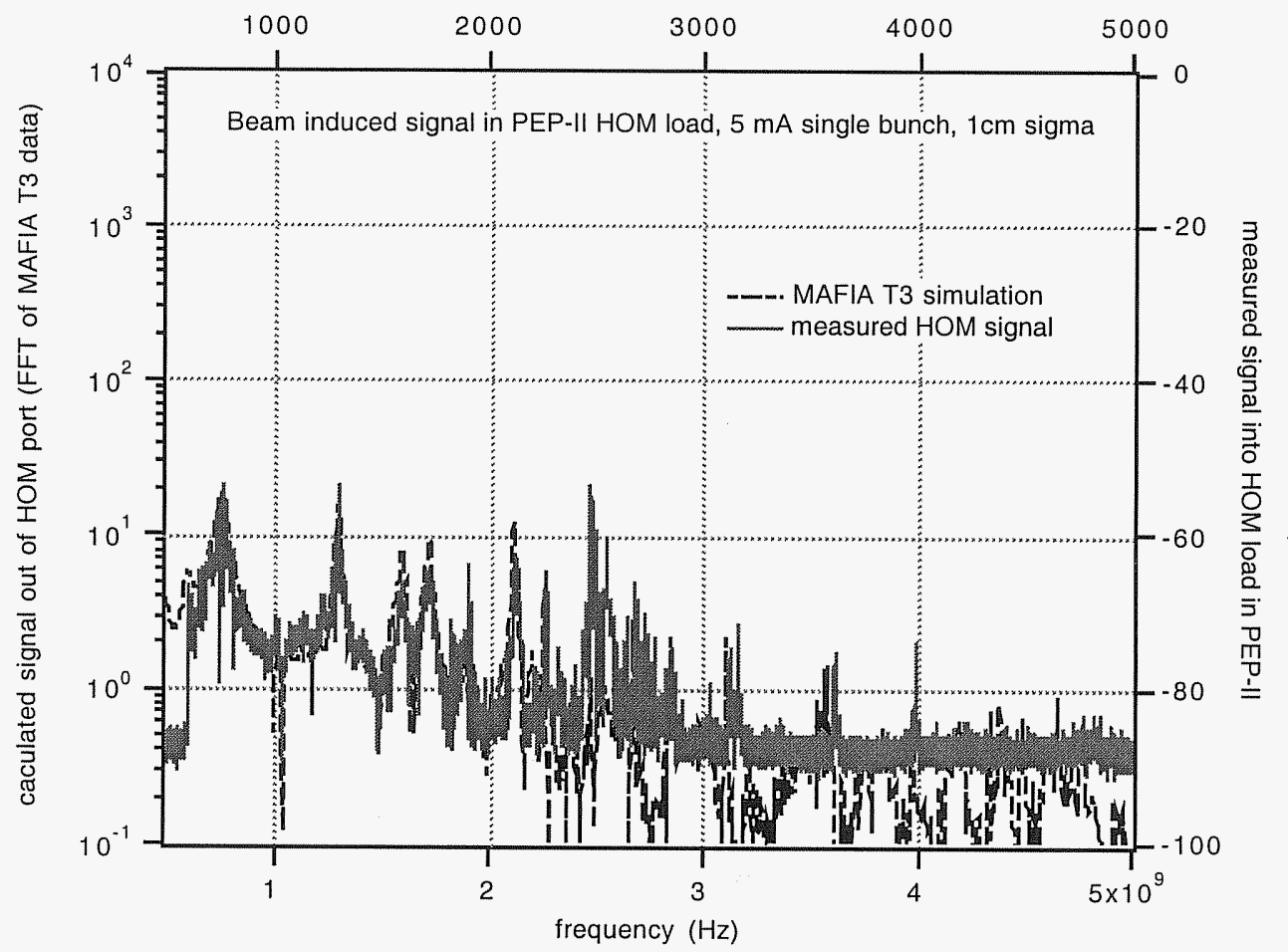

Figure 4. Calculated mode spectrum reaching the HOM load compared with signal measured with beam, including frequencies above cutoff

Figure 4 shows the spectrum extending out to frequencies above cut-off. The noise floor of the spectrum analyzer can be clearly seen in the measured data at high frequency but the peaks still correspond well with those in the calculated spectrum. Also, there is a range just above cut-off where the measured signal appears to exceed that expected from the cavity modes, which may be due to energy propagating into the cavity down the beam pipe from nearby discontinuities in the vacuum chamber. The close correlation of these signals and between the calculated and measured mode strengths gives confidence in the broadband simulation method as a useful tool for optimizing the overall geometry. 
Mechanical design, stresses and fabrication

While the PEP-II cavity, figure 5, is a very successful design it is a rather expensive assembly to make and contains some features and uses some processes that may be improved upon, such as the use of pure copper plating on the body to allow a subsequent brazing step (which was eventually eliminated from the design). Lessons learned during the fabrication [11], can be applied to future projects. Some of these ideas were applied to the six cavities built for the ALS third harmonic RF system. The thermal and stress analysis of the PEP-II cavity showed high temperature and stress around the HOM irises due to the tight radii of curvature at the ends of the slots and in the blend between the slots and the body. Careful arrangement of the cooling channels and increasing the blend radius mitigated the problem to a safe level for use in PEP-II but it is prudent to revisit this issue for future designs. Simple changes in this area may reduce the RF current concentration and therefore lower the temperature and stress without reducing the effectiveness of the HOM damping scheme.

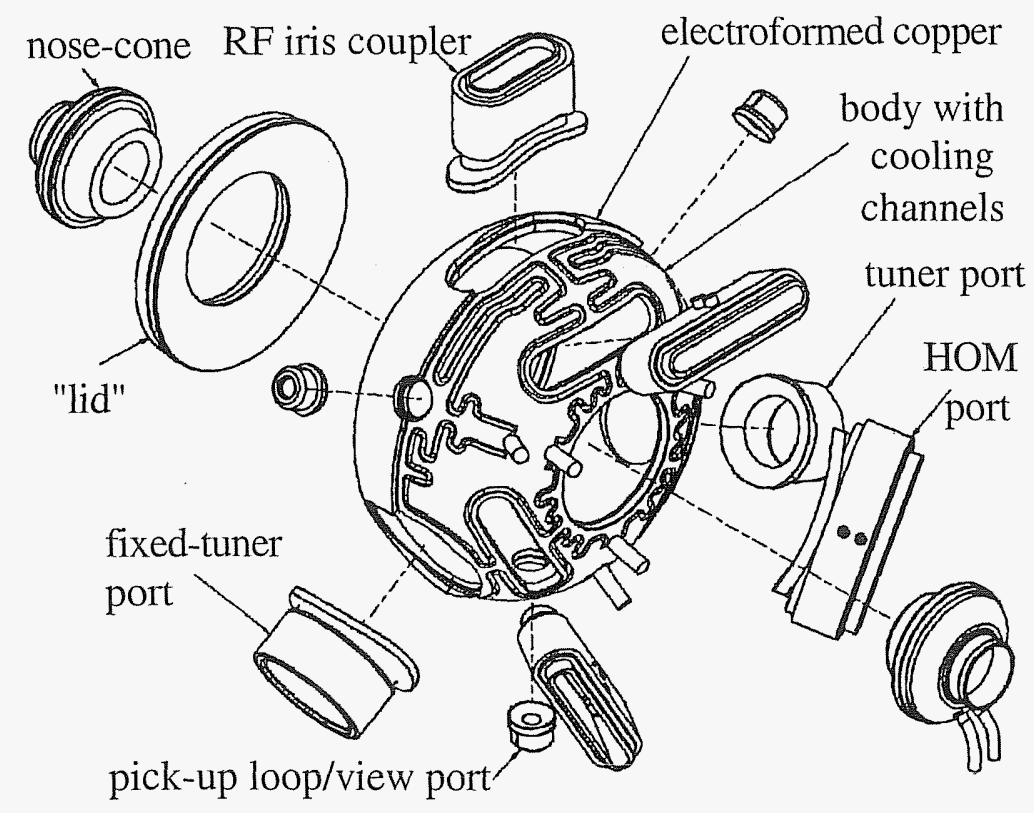

Figure 5. PEP-II cavity major components (minus flanges). 


\section{PEP-II cavity analysis}

The RF losses for the PEP-II shape were originally calculated using a purpose written code based on a boundary integral method (BIM), [12], (which is unfortunately no longer available), and transferred to ANSYS [13], for the thermal and stress calculations by the finite element. method (FEM). The peak value was about 120 $\mathrm{W} / \mathrm{cm}^{2}$ without the coupling port, see figure 6 , but dropped to about $106 \mathrm{~W} / \mathrm{cm}^{2}$ with the racetrack coupler port added. (Note that in the earliest design the iris had a 1/4" blend radius and the peak power loss was over $171 \mathrm{~W} / \mathrm{cm}^{2}$ ).

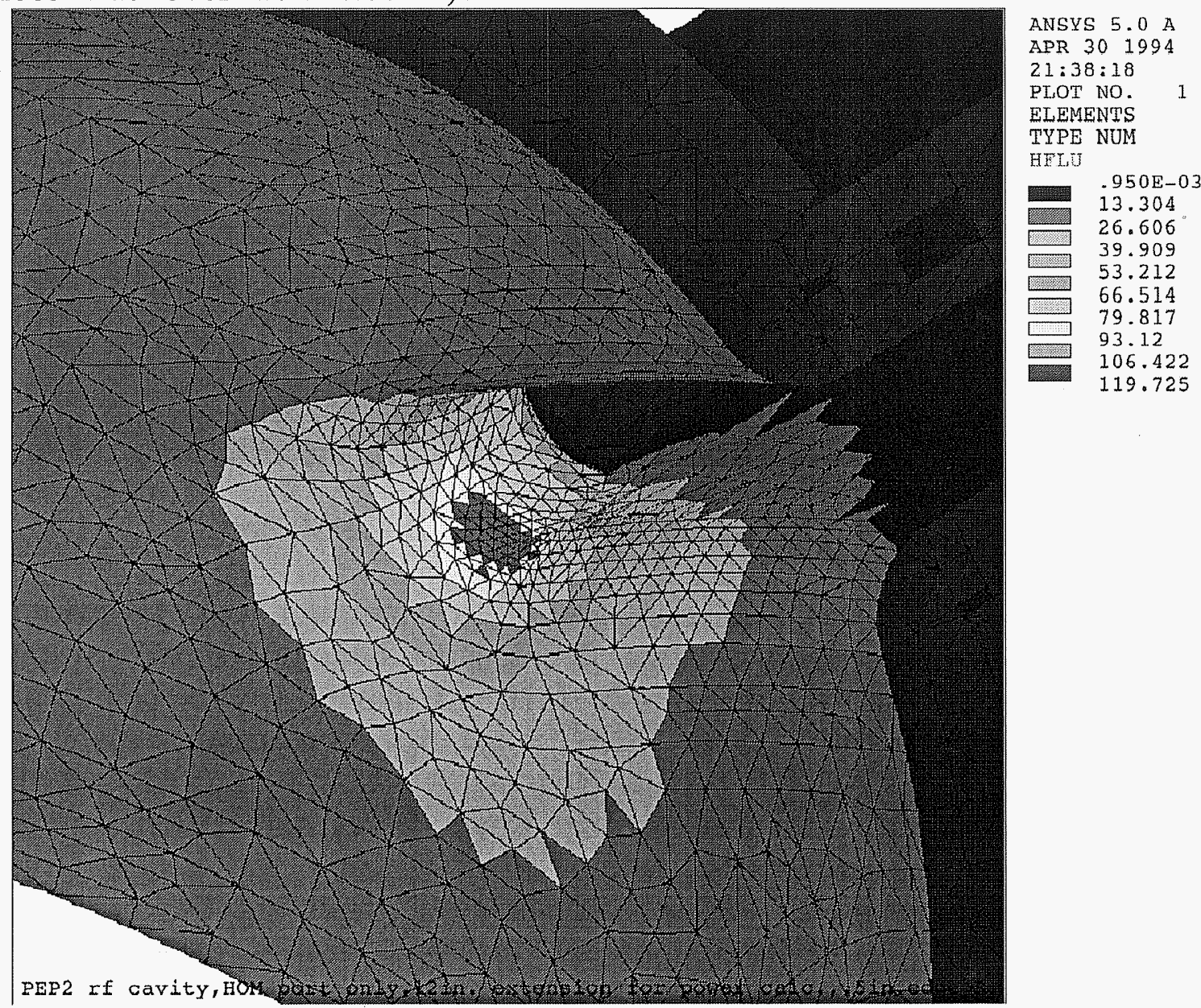

Figure 6. Original Chalk River BIM results (displayed in ANSYS).

It is now possible to calculate the RF losses directly in ANSYS with the addition of a new high frequency electromagnetic element type [14]. As a way to verify the new method the PEP-II design was re-analyzed using a completely new mesh and RF losses calculated within ANSYS. At the same time the RF losses were also computed using the new Omega3p 3D code at SLAC [15]. Omega3p is a parallel 
distributed memory finite-element code for solving electromagnetics in the frequency domain for large complex three-dimensional geometries. Omega3P combines an unstructured mesh, adaptive mesh refinement, high fidelity geometry representations, and a quadratic finite-element formulation to obtain high accuracy. The code uses domain decomposition over multiple processors and a superior algorithm for the generalized eigenvalue problem to achieve scalable performance. On the Cray T3E at NERSC, Omega3P has solved problem sizes in the tens of millions of degrees of freedom.

PEP-II results, ANSYS:

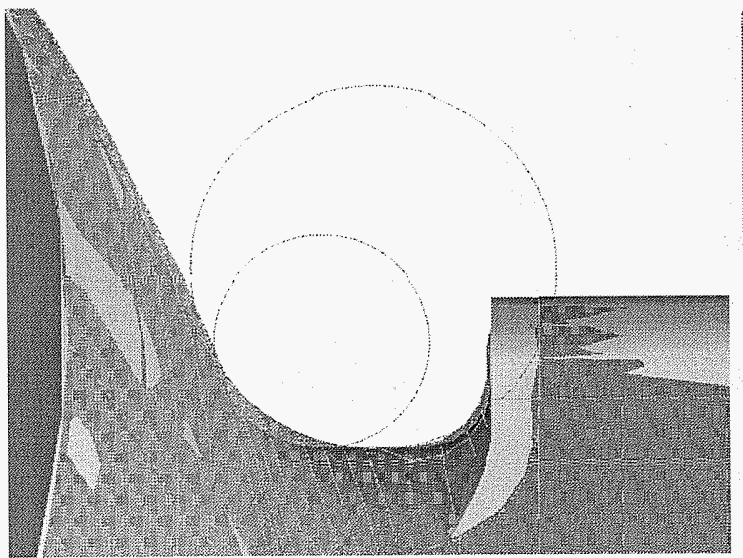

a

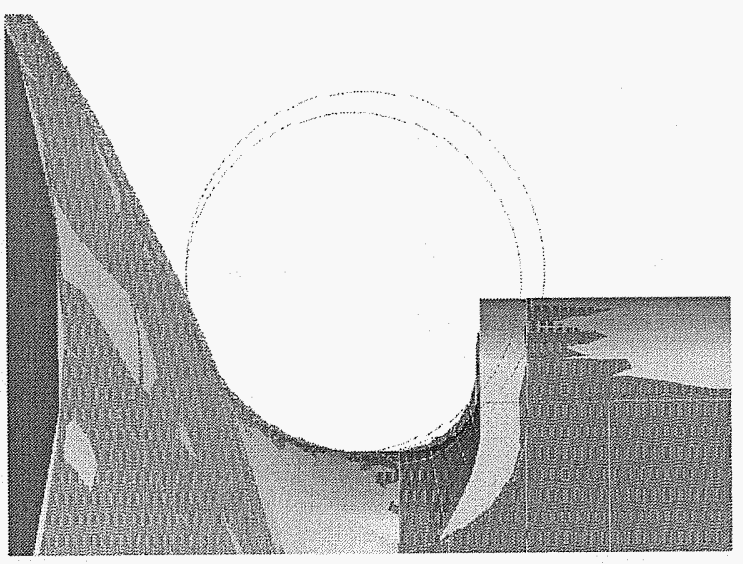

b

Figure 7. Radius at Base of PEP-II HOM Port.

In order to validate our ability to conduct RF frequency, thermal, and stress analyses, we decided to benchmark our system by analyzing the known PEP-II $476 \mathrm{MHz}$ RF cavity. This cavity has been analyzed previously at SLAC, and its performance is well known. Recent SLAC RF calculations using ANSYS showed that the exact same mesh as was used for the Chalk River calculation resulted in a power loss distribution which differed from the Chalk River result by $0.5 \%$ at most. After independently modeling the cavity at LBNL, however, it became apparent that the design drawings and shape that was built reflected a slightly different geometry in some details than was analyzed during its design. In particular, the blended radius at the base of the HOM port, which is $1 / 2$ " at the symmetry plane in both models, grew with distance from the symmetry plane in the SLAC model. The SLAC model had been constructed entirely in ANSYS, with the 1/2" radius seen in figure 6 at the cut plane extended around the HOM port base by intersecting a series of toroidal and racetrack-shaped surfaces. The LBNL model 
was imported into ANSYS with a constant blend radius at the HOM port base. Figure 7 a shows the design shape in pink, with the analyzed shape overlaid in transparent blue, with the circles representing the approximate curvatures of the fillets. In figure $7 \mathrm{a}$ the difference in HOM radii can be seen; the design shape is approximately $1 / 2 "$ in radius, while the SLAC model shape is approximately $3 / 4 "$. In order to quantify the difference due to this discrepancy, two models were constructed, one with the design radius, 1/2", while the other model, figure $7 \mathrm{~b}$, had a $3 / 4 "$. blended radius at the base of the HOM port.

Figure 8 shows the shape that was originally analyzed, with the larger radius, while the design shape, with the $1 / 2$ " radius, is shown in figure 9. These figures illustrate the heat flux in the cavity wall due to losses from the RF currents. Both models are normalized to $150 \mathrm{~kW}$ dissipation per full cavity, or $25 \mathrm{~kW}$ per sixth. In the cavity with the larger radius, the maximum heat flux is $118 \mathrm{~W} / \mathrm{cm}^{2}$, which is between the previously calculated values with and without the racetrack coupler port. Mesh refinement studies in the 'hot' area showed that the previously calculated maximum heat flux values may have been as much as $6 \%$ low due to the coarseness of the original mesh in that area. In the design cavity the heat flux is higher, at approximately $133 \mathrm{~W} / \mathrm{cm}^{2}$. This behavior is consistent with the fact that the HOM radius is critical in determining the local heat flux; the smaller radius has a higher heat flux density. The larger radius concentrates the heat less and the peak is comparable to that on the back of the iris where the field is lower but the radius is smaller. 


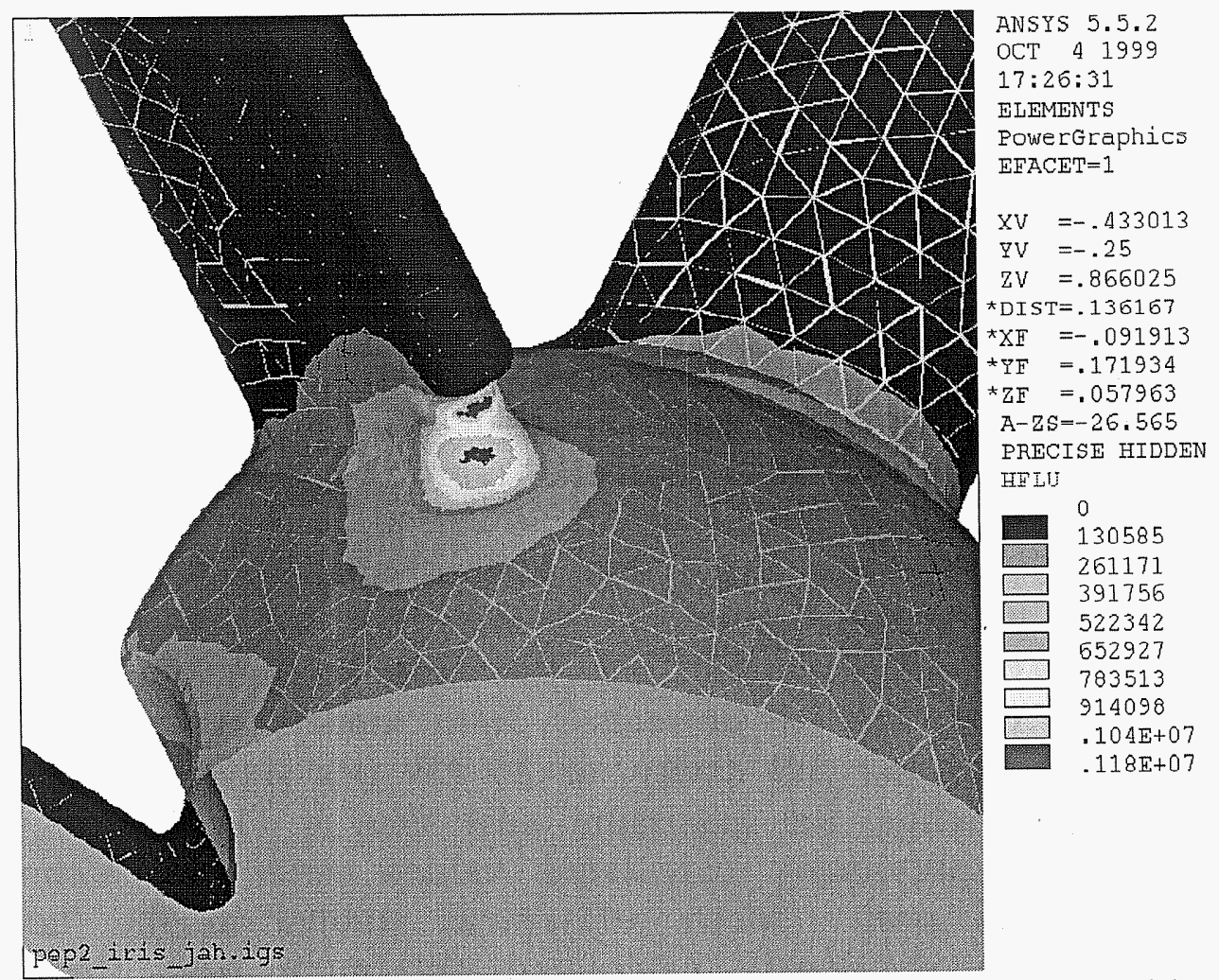

Figure 8. Heat flux in PEP-II cavity with larger HOM port blended radius.

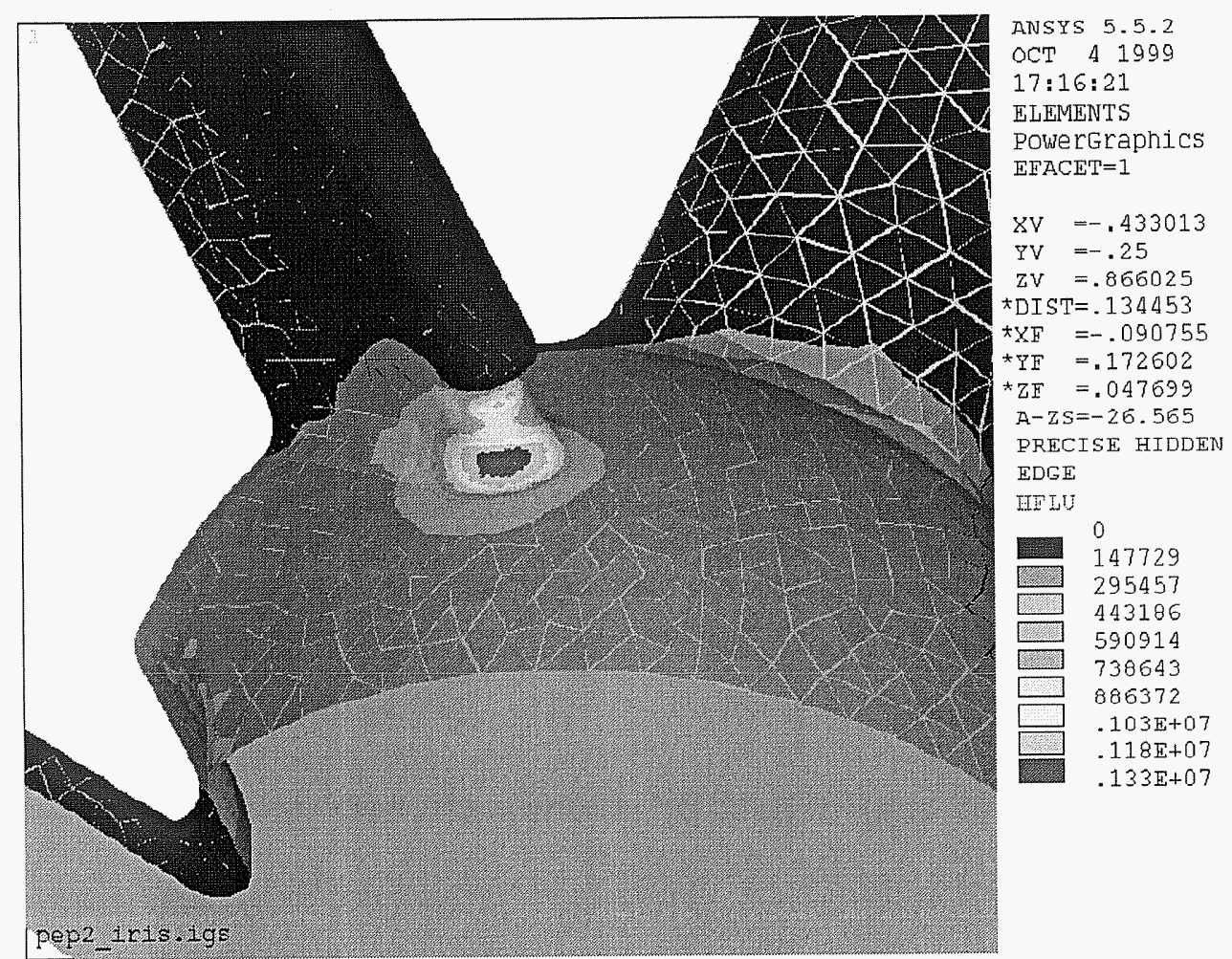

Figure 9. Heat flux in PEP-II cavity as built 


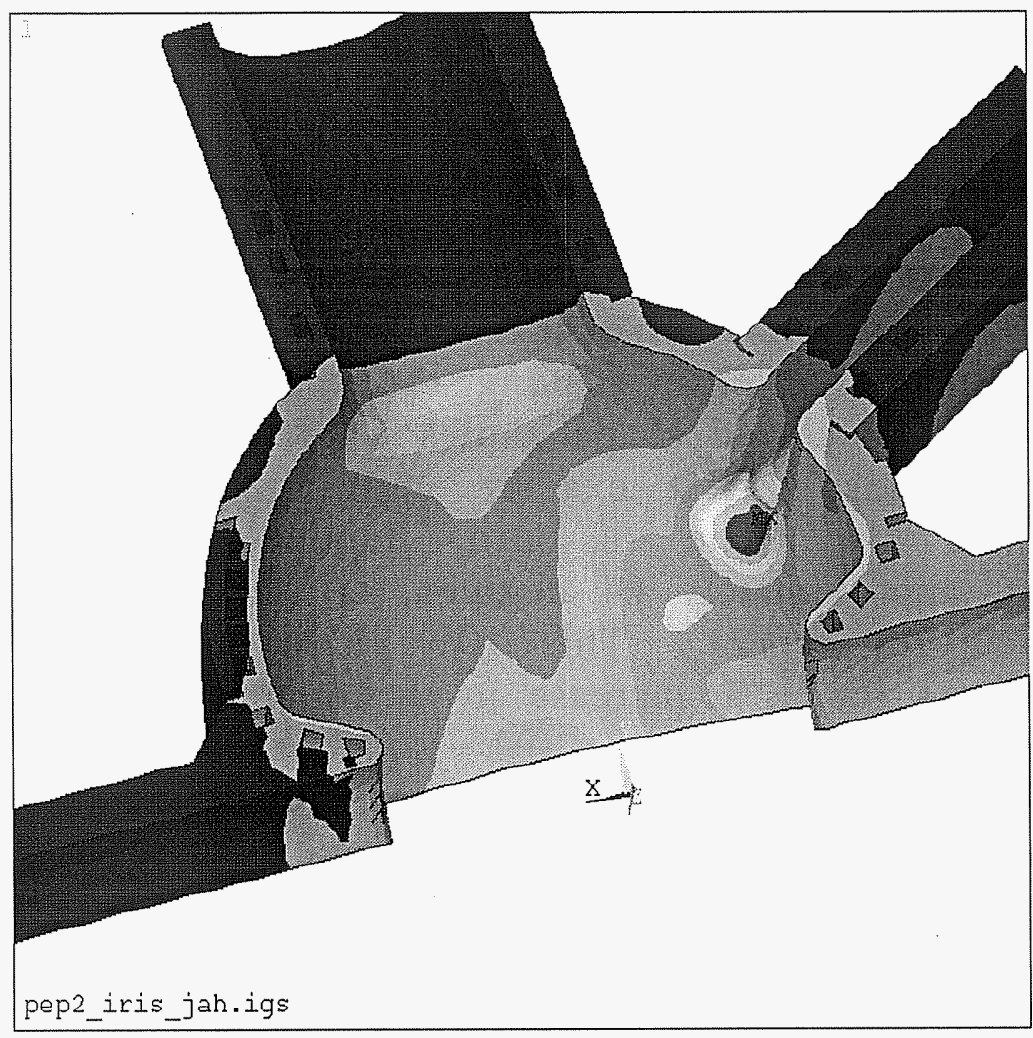

ANSYS 5.5 .2

AUG 271999

$11: 04: 40$

NODAI SOLUTION

$S T E P=1$

$\mathrm{SUB}=1$

TIME $=1$

TEMP

(AVG)

RSYS $=0$

PowerGraphics

EFACET $=1$

AVRES $=$ Mat

$\operatorname{SMX}=58.26$

0
6.473

12.947

19.42

25.893

$\square \quad 32.366$

38.84
$\square$ 4.313

51.786

pep2_iris jah.igs

Figure 10. Temperature distribution in cavity with larger HOM port base radius.

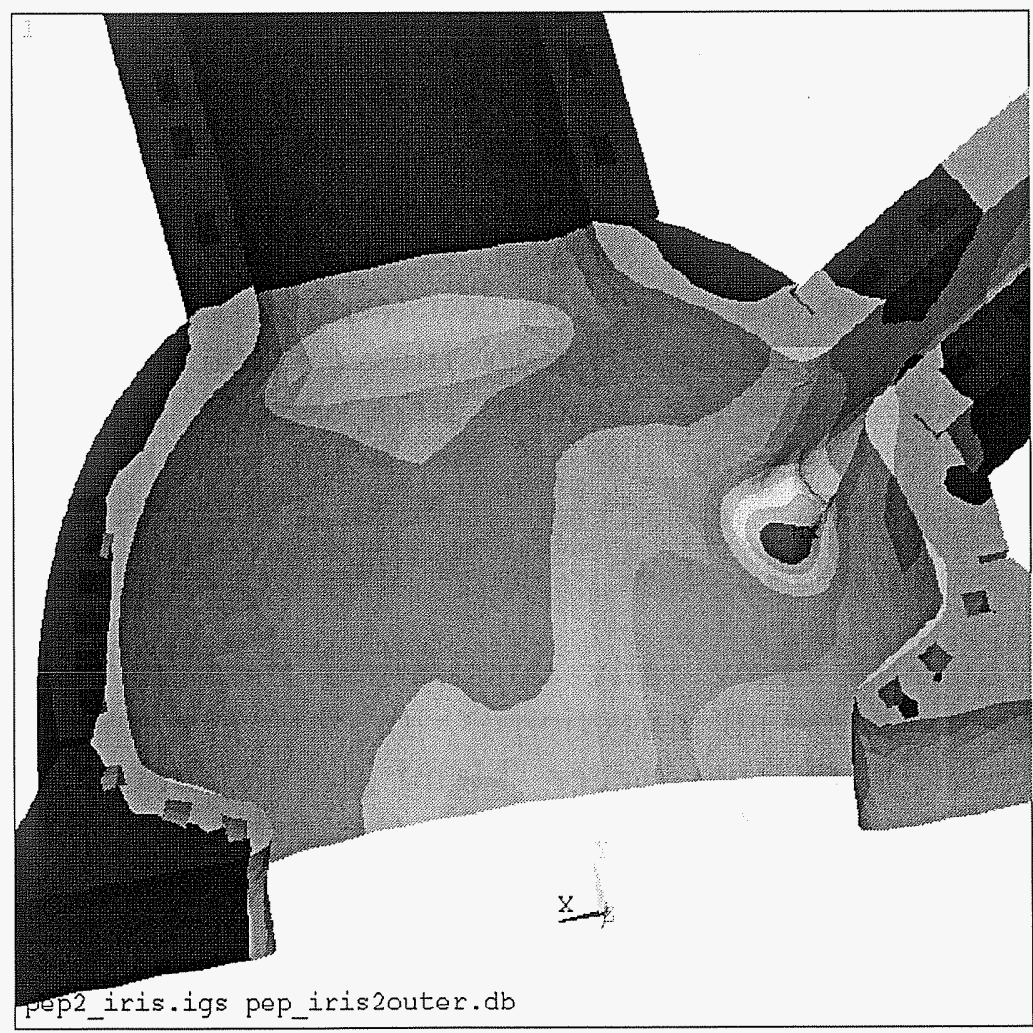

ANSYS 5.5 .2

AUG 301999

$10: 42: 58$

NODAL SOLUTION STEP $=1$

SUB $=1$

FREQ $=1$

TEMP

(AVG)

RSYS=0

PowerGraphics

EFACE $T=1$

AVRE $S=$ Mat

SMN $=.129409$

SMX $=64.009$

.129409

7.227

14.325

21.423

28.52

35.518

35.518

49.814

56.911

64.009

Figure 11. Temperature distribution in cavity, as built. 
The temperature distribution in the PEP-II Cavity under nominal cooling is shown in figure 10 for the HOM with the larger blend radius and figure 11 for the design shape, as built. In each model the cooling channels (some of which can be seen crossing the symmetry line of the model) are constrained with convection coefficients of $18,000 \mathrm{~W} / \mathrm{m}^{2}{ }^{\circ} \mathrm{C}$ and bulk temperatures of $0{ }^{\circ} \mathrm{C}$. Thus, both plots show temperature rise above water temperature. The effect of water temperature change along the channel (5-6 degrees), is not included. The analyzed shape shows a 58 degree temperature rise, which corresponds almost exactly with the original results achieved at SLAC [16]. The "as-built" model shows a larger temperature range, approximately 64 degrees, or $10 \%$ higher than in the previously analyzed model. This $10 \%$ increase in temperature corresponds closely to the $12 \%$ increase in maximum heat flux shown in the previous figure.

Figures 12 and 13 show the stresses calculated when the temperature distributions from the thermal models were applied to the full cavity body with free constraints at the port boundaries. In both models the right beam port is fixed, while the left beam port is free in the beam direction. The HOM and coupling ports are both free. The undisplaced shape is shown as a faint line overlaid on the contour plot. The model with the larger blend radius, figure 12 , shows a maximum stress of $69 \mathrm{MPa}$, or approximately 10,000 Psi, which corresponds almost exactly to numbers calculated at SLAC. The design model, figure 13 , shows a stress that is approximately $10 \%$ higher, at $75 \mathrm{MPa}$, corresponding to the $10 \%$ increase in temperature. Atmospheric loading is also applied to both models, but its contribution to the overall stresses is minimal.

In order to compute the effect of constraining the coupler port, the original free models were rerun with full constraints on the end of the coupler. Constraining the models here caused the maximum stress location to shift from one side of the HOM port to the center, and the maximum stress increased by $25 \%$ in the model with the larger blend radius, figure 14 , and $13 \%$ in the design cavity, figure 15 .

Figures 16 and 17 show fully constrained models, with HOM and coupler ports fixed in all directions. In this extreme case the stress in the model with the larger blend radius increases to a maximum of $140 \mathrm{MPa}(20,000 \mathrm{Psi})$, while the design model shows $10 \%$ higher stresses, at $156 \mathrm{MPa}(22,000 \mathrm{Psi})$. The $10 \%$ increase from analyzed to design models is again observed, as would be expected with stresses that are due solely to thermal expansion. Atmospheric loading is applied to both models, but is once again negligible. 
These analyses show that the port constraints are of high importance in the overall structural performance of the cavity. Care must be taken to decouple the waveguides and HOM loads in the real installation. However, notice that the maximum deflection in the unconstrained case is only about .1 mm (DMX in figure 13). A physical support which flexes only a small amount under the force of the expanding cavity will relieve a significant part of this extra stress. 


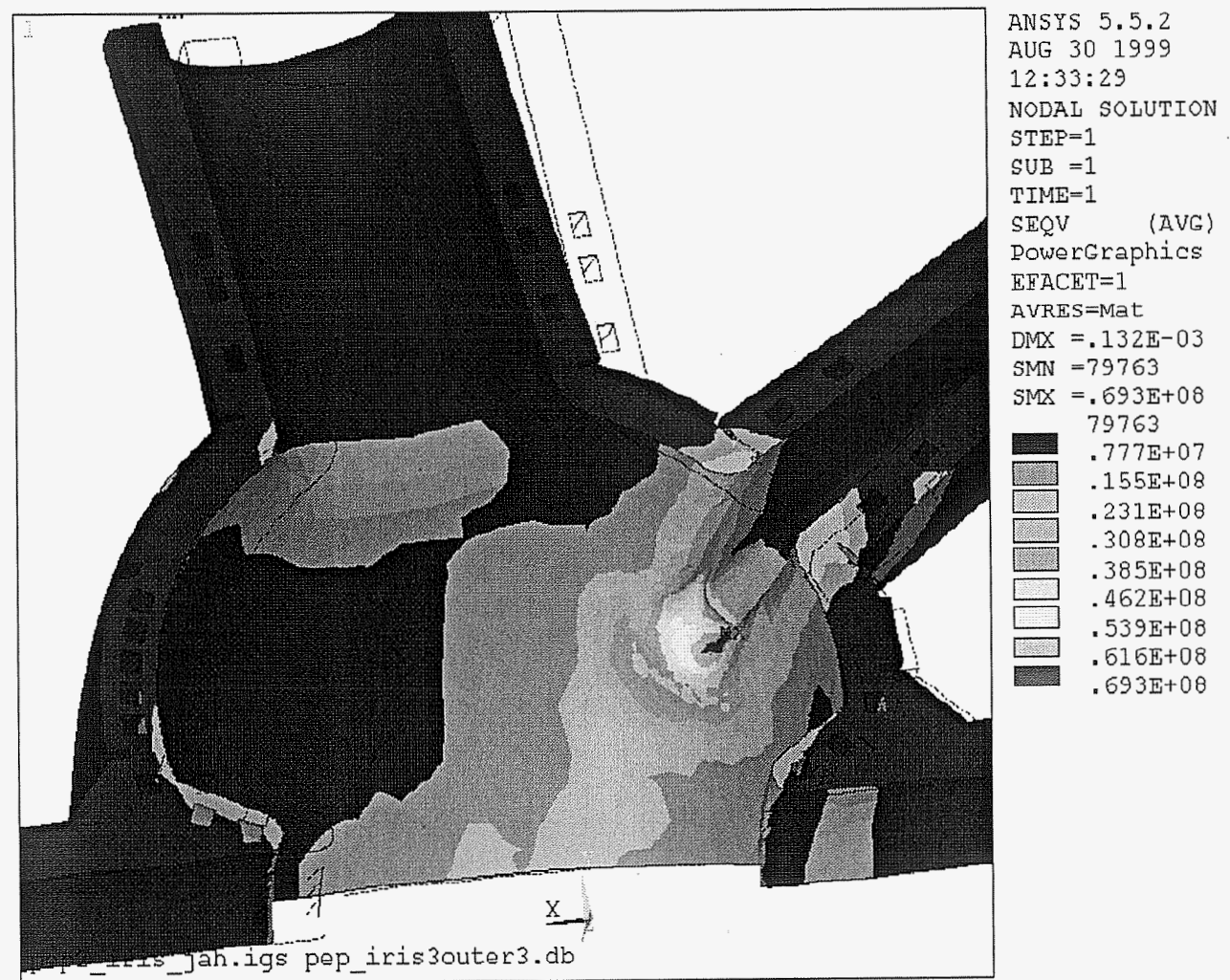

Figure 12. Thermal stresses, ports unconstrained, with larger HOM port base radius.

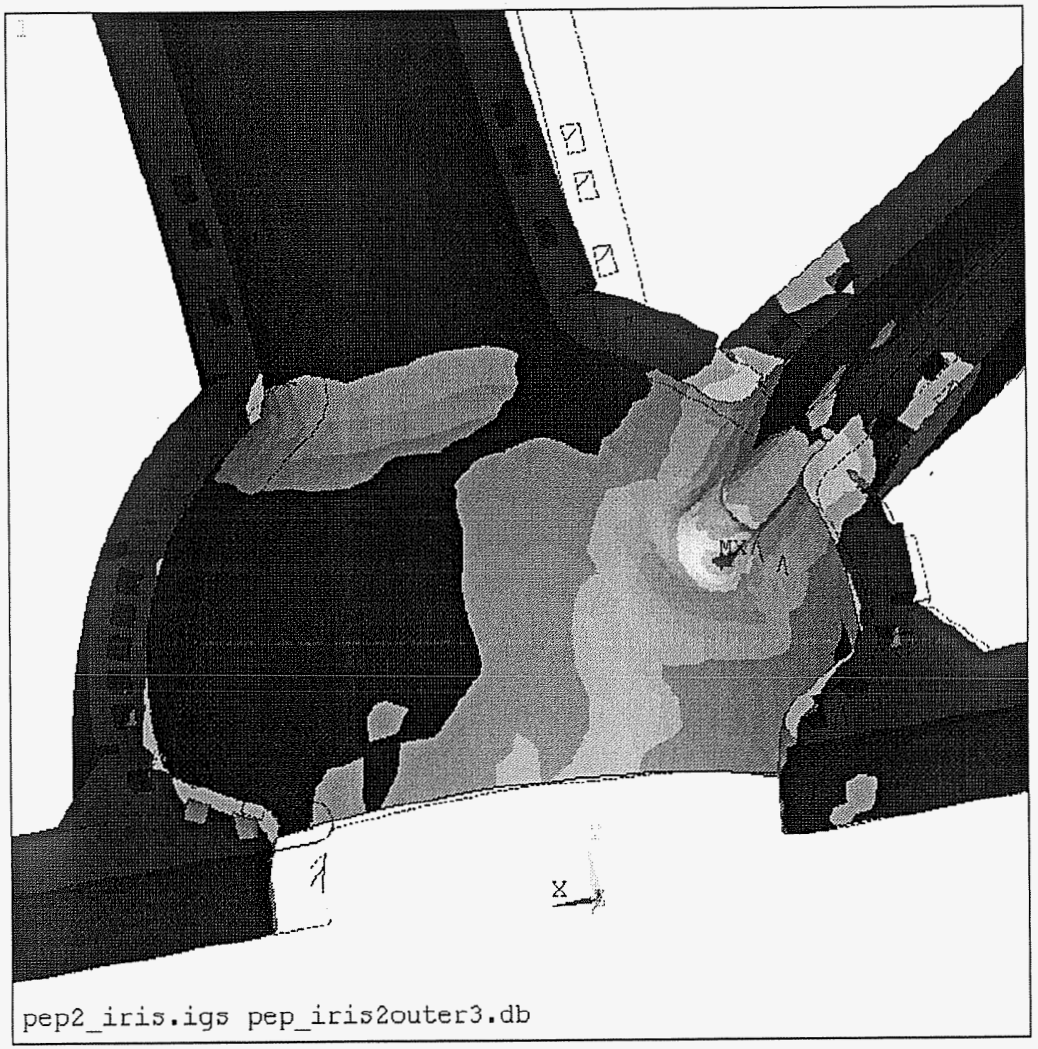

ANSYS 5.5 .2

AUG 301999

$11: 49: 17$

NODAL SOLUTION

STEP $=1$

SUB $=1$

TIME $=1$

SEQV (AVG)

PowerGraphics

EFACET $=1$

AVRES=Mat

$\mathrm{DMX}=.144 \mathrm{E}-03$

SMN $=60292$

$5 M S=.751 \mathrm{E}+08$

60292

$.839 \mathrm{E}+07$

$.167 \mathrm{E}+08$

$.251 \mathrm{E}+08$

$.334 \mathrm{E}+08$

$.417 \mathrm{E}+08$

$.501 \mathrm{E}+08$

$584 \mathrm{E}+08$

$.667 \mathrm{E}+08$

$.751 \mathrm{E}+08$

Figure 13. Thermal stresses, ports unconstrained, as built. 


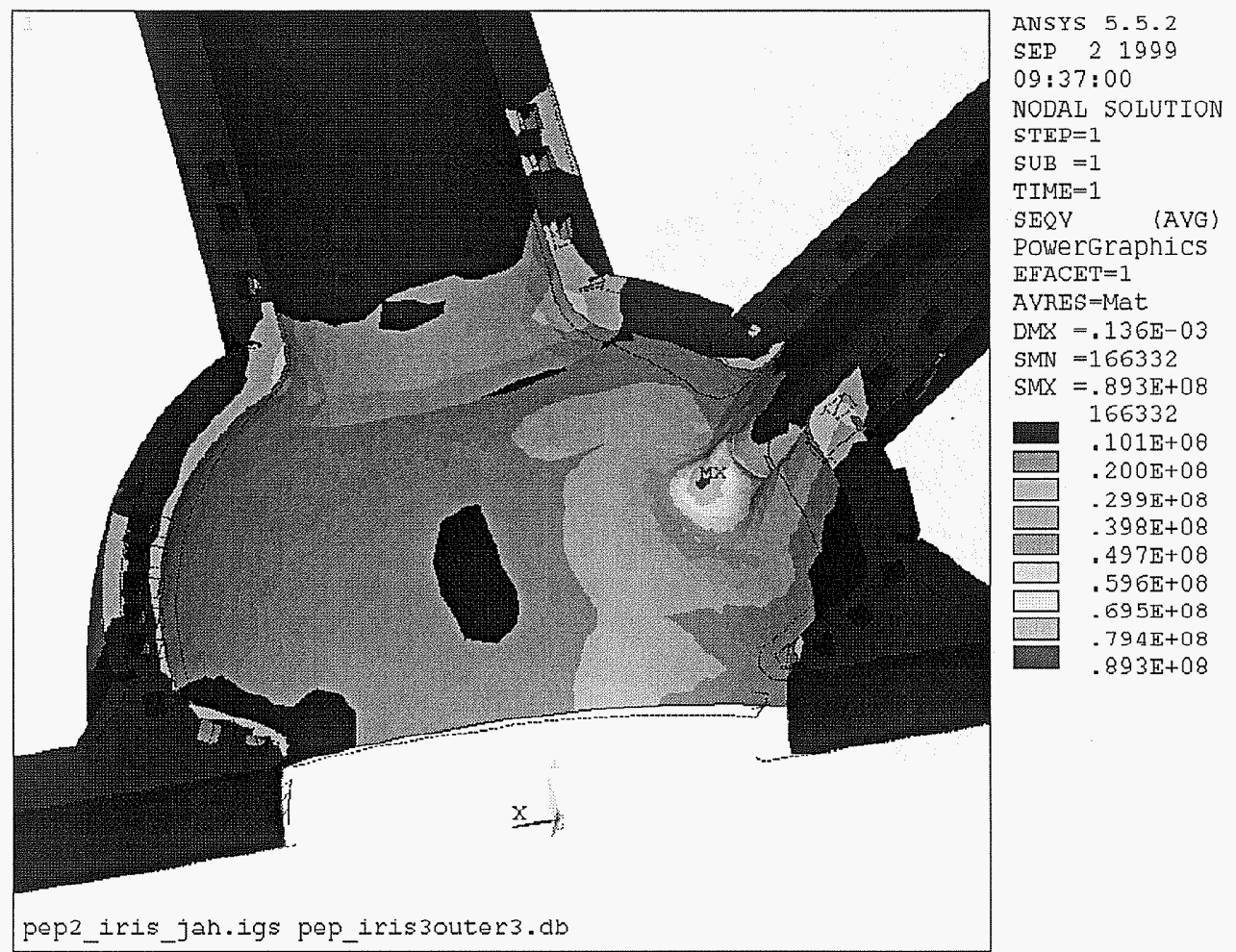

Figure 14. Thermal stresses, coupler port fixed, with larger HOM port base radius.

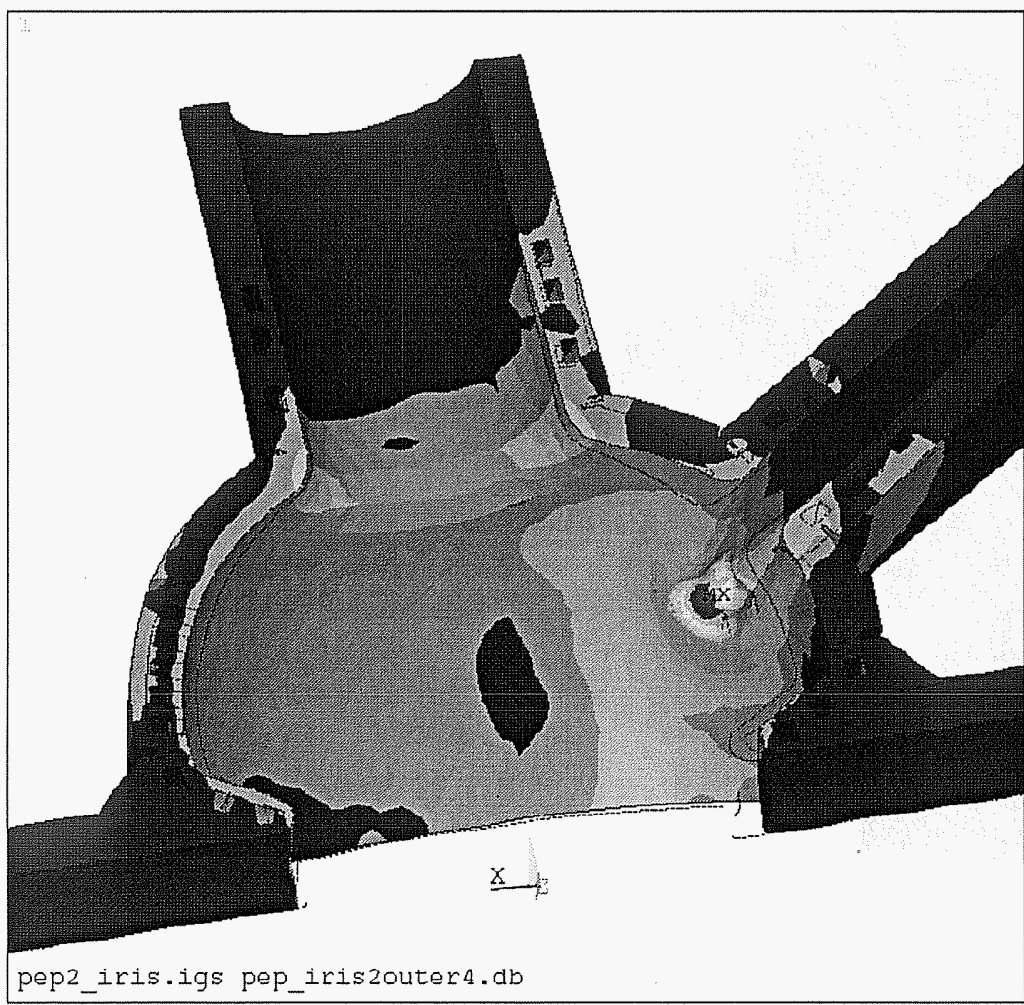

ANSYS 5.5 .2

SED 11999

20:00:57

NODAI SOLUTION

STEP $=1$

SUB $=1$

TIME $=1$

SEQV

(AVG)

Powergraphics

EFACET=1

AVRES=Mat

$\mathrm{DMX}=.158 \mathrm{E}-03$

SMN $=15809$

SINX $=.846 E+08$

158094

[. $.954 \mathrm{E}+07$

$.189 \mathrm{E}+08$

$.283 \mathrm{E}+08$

$.377 \mathrm{E}+08$

$.471 \mathrm{E}+08$

$.564 \mathrm{E}+08$

$.558 \mathrm{E}+08$

$.752 \mathrm{E}+08$

$.846 \mathrm{E}+08$

Figure 15. Thermal stresses, coupler port fixed, as built. 


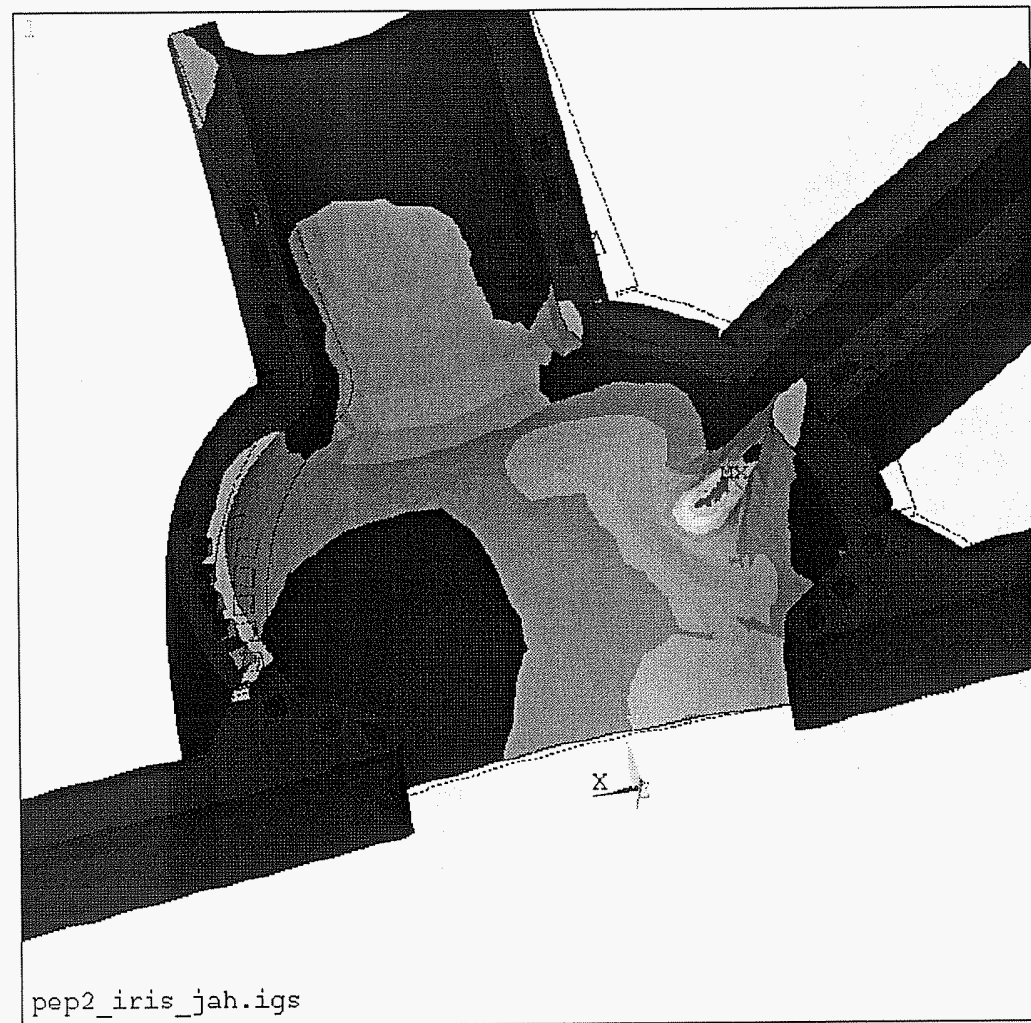

ANSYS 5.5 .2

AUG 271999

$11: 01: 39$

NODAI SOLUTION

$S T E P=1$

SUB $=1$

TIME $=1$

SEQV

(AVG)

Dowergraphics

EFACET $=1$

AVRES $=$ Mat

$\mathrm{DMX}=.104 \mathrm{E}-03$

SMN $=342759$

$\operatorname{SMX}=.141 \mathrm{E}+09$

342759

$.159 \mathrm{E}+08$

$.315 E+08$

$471 \mathrm{E}+08$

$.626 \mathrm{E}+08$

$.782 \mathrm{E}+08$

$.938 \mathrm{E}+08$

$.109 \mathrm{E}+09$

$.125 \mathrm{E}+09$

$.141 \mathrm{E}+09$

Figure 16. Thermal stresses, fully constrained cavity model, with larger HOM port base radius.

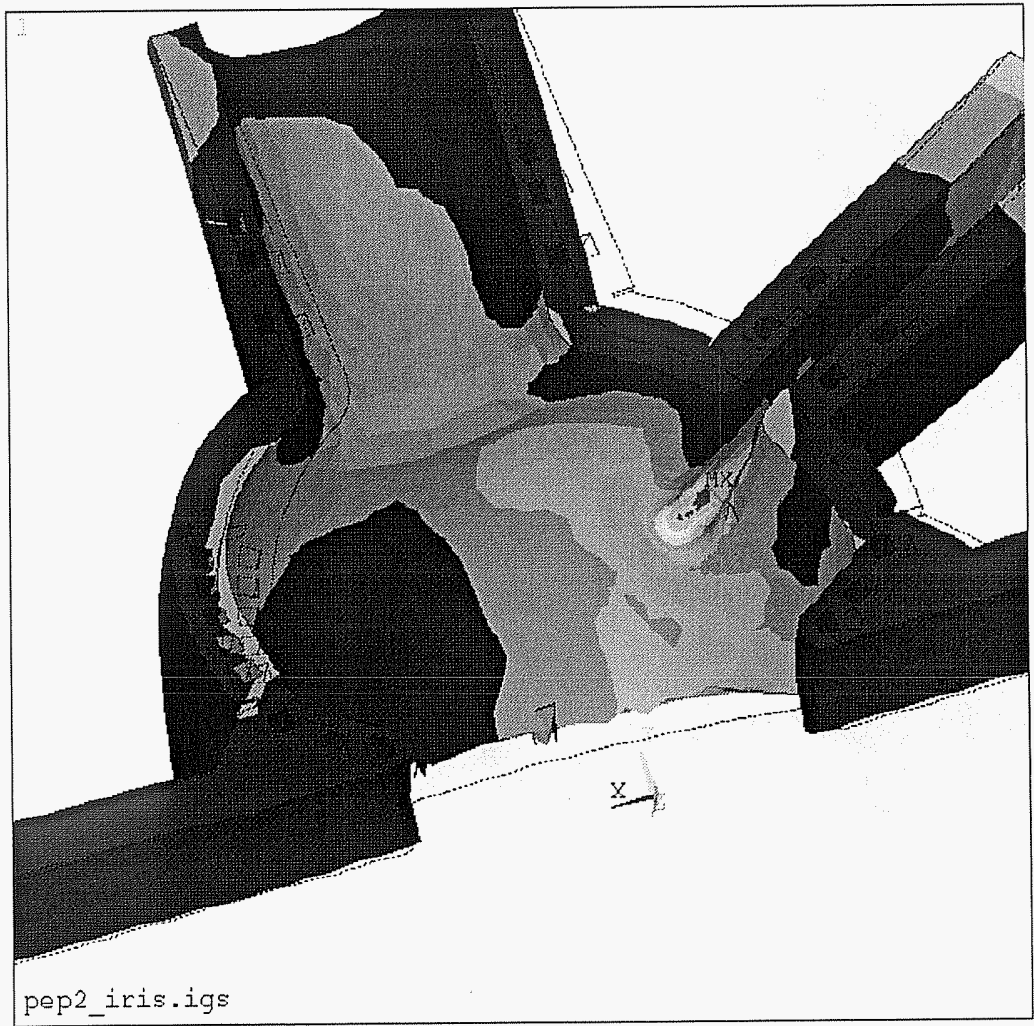

ANSYS 5.5 .2

OCT 61999

$09: 30: 04$

NODA. SOLUTION

STEF $=1$

SUB $=1$

FREQ $=1$

SEQV

(AVG)

powergraphics

EFACET $=1$

AVRES = Mat

$\mathrm{DMX}=.122 \mathrm{E}-03$

$5 M N=562082$

$\operatorname{SMX}=.156 \mathrm{E}+09$

562082

$.178 \mathrm{E}+08$

$.351 \mathrm{E}+08$

$.524 \mathrm{E}+00$

$.696 \mathrm{E}+08$

$.869 \mathrm{E}+08$

$.104 \mathrm{E}+09$

$.121 \mathrm{E}+09$

$.139 \mathrm{E}+09$

$.156 \mathrm{E}+09$

Figure 17. Thermal stresses, fully constrained cavity model, as built. 
PEP-II results, Omega:

Omega3P is a parallel distributed memory finite-element eigensolver for finding resonant modes in large, three-dimensional RF cavities of complex shape. The code inputs the cavity geometry directly from CAD tools, and uses quadratic elements and NURBS representation to conform to curved surfaces. It partitions the mesh onto multiple processors by domain decomposition, and applies an efficient parallel algorithm to solve the resulting generalized eigenvalue problem with fast convergence and near linear speedup. Using 128 processors on the SGI/CRAY T3E at NERSC, Omega3P solves a problem with 1 million degrees of freedom to $10 \mathrm{e}-10$ accuracy in the eigenvalue after only 4 iterations and consumes less than 8 minutes of CPU time.

Omega3p has been used to analyze the PEP-II cavity in several different runs. Figure 18 shows the result with an unstructured mesh of approximately uniform density with $943 \mathrm{~K}$ tetrahedral elements. This model was run using the quadratic element formulation, the results are shown in table 3. To further improve the accuracy the mesh was refined locally around the hot spot, and relaxed elsewhere to reduce the problem size. Figures 19 to 21 show the meshes and the heat flux solutions in the area of the hot spot. The body mesh is slightly coarser than the uniform case but the local density around the hot spot was increased in stages, from an average element size of $5 \mathrm{~mm}$ to only $1.5 \mathrm{~mm}$. The resulting $\mathrm{RF}$ heat loss density, tables 4 to 6 , rapidly converges to a stable number of around $139 \mathrm{~W} / \mathrm{cm}^{2}$. The ANSYS results agree quite well with this value. All of the converged results show the main hot spot on the nosecone side of the iris and the smaller, secondary hot spot on the top side of the iris. 


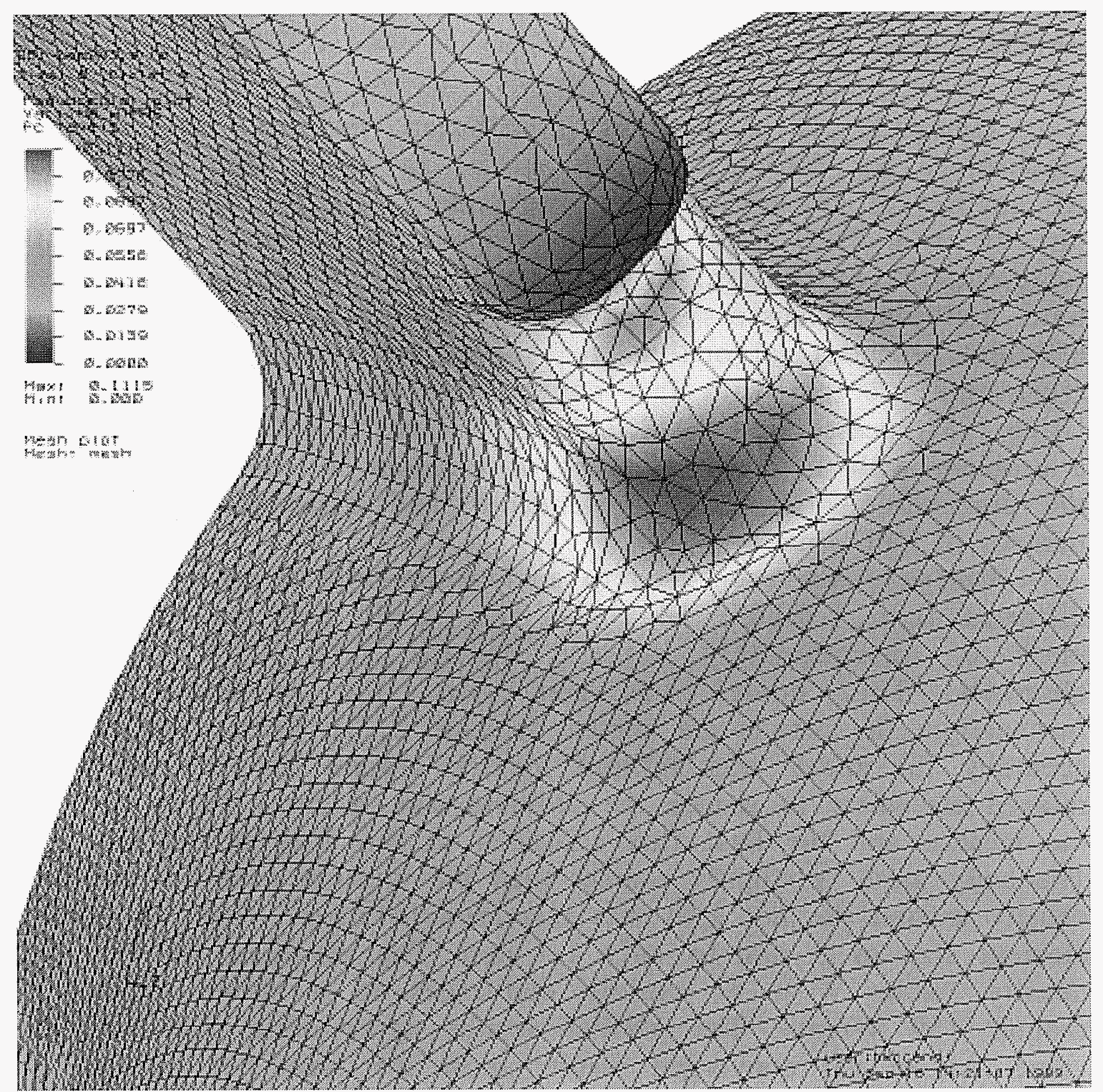

Figure 18. Wall loss calculated by Omega3p for PEP-II geometry, uniform mesh density.

Table 3. Omega3p results for uniform mesh

\begin{tabular}{|c|c|}
\hline & $943 \mathrm{~K}$ Quadratic \\
\hline frequency $(\mathrm{MHz})$ & 476.514 \\
\hline degrees of freedom & 5971310 \\
\hline peak power $\mathrm{W} / \mathrm{cm}^{2}$ & 135.1 \\
\hline
\end{tabular}

* normalized to $150 \mathrm{~kW}$ total wall loss 


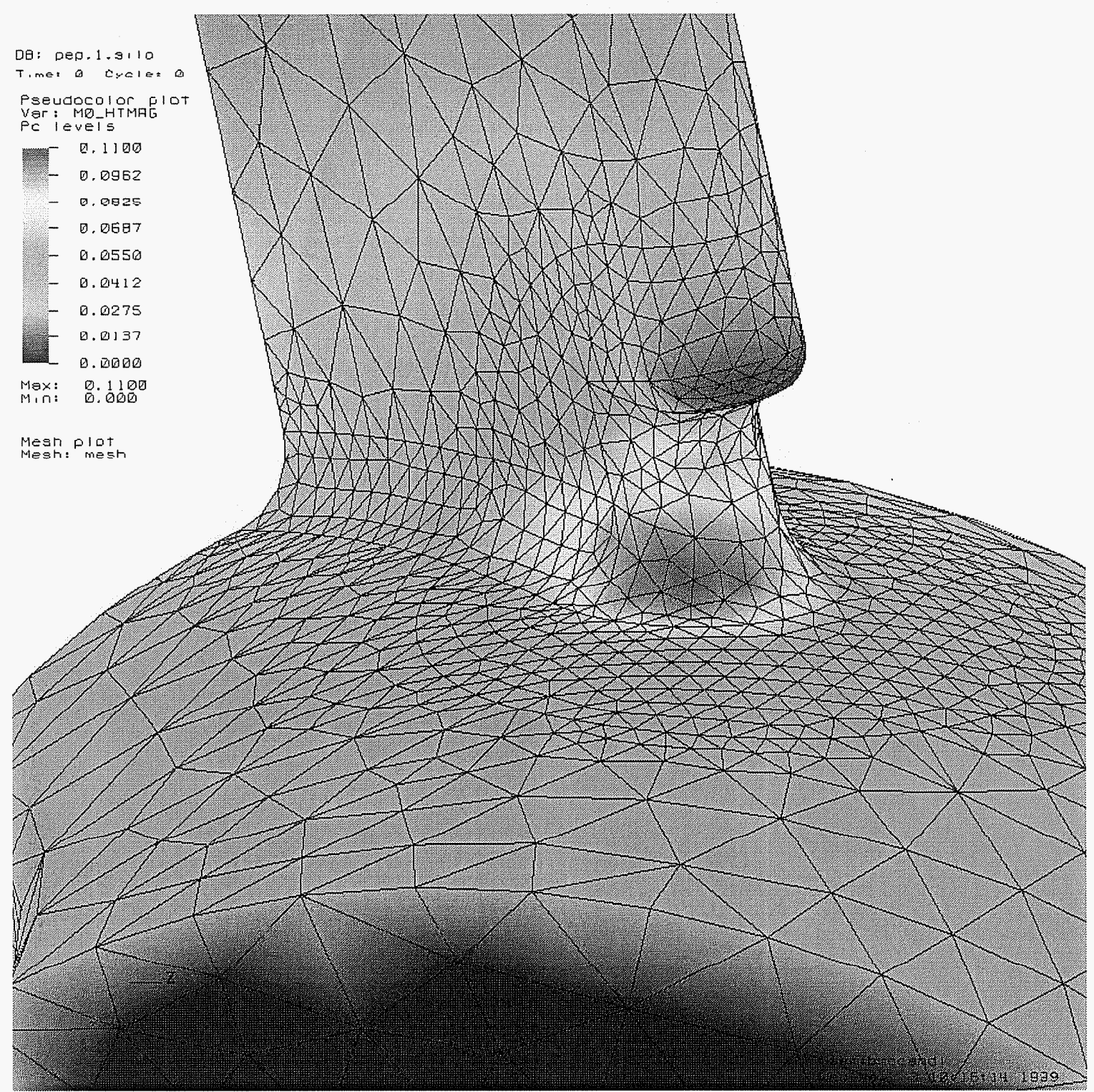

Figure 19. PEP-II model with $5 \mathrm{~mm}$ local element size.

Table 4. PEP-II results with $5 \mathrm{~mm}$ local element size

\begin{tabular}{|l|l|}
\hline mesh size (hot spot): & $5 \mathrm{~mm}$ \\
\hline mesh size (nose): & $5 \mathrm{~mm}$ \\
\hline mesh size (everywhere else): & $18 \mathrm{~mm}$ \\
\hline \# elements: & 23390 \\
\hline \# degrees of freedom: & 142914 \\
\hline frequency: & 477119466 \\
\hline Peak power density & $6.81196 \mathrm{e}-07 \mathrm{~W} / \mathrm{m}^{\wedge} 2$ \\
\hline total power (1/6 model) & $3.49067 \mathrm{e}-05 \mathrm{~W}$ \\
\hline Peak power density* & $1.2811 \mathrm{MW} / \mathrm{m}^{\wedge} 2$ \\
\hline
\end{tabular}

* normalized to $150 \mathrm{~kW}$ total wall loss 


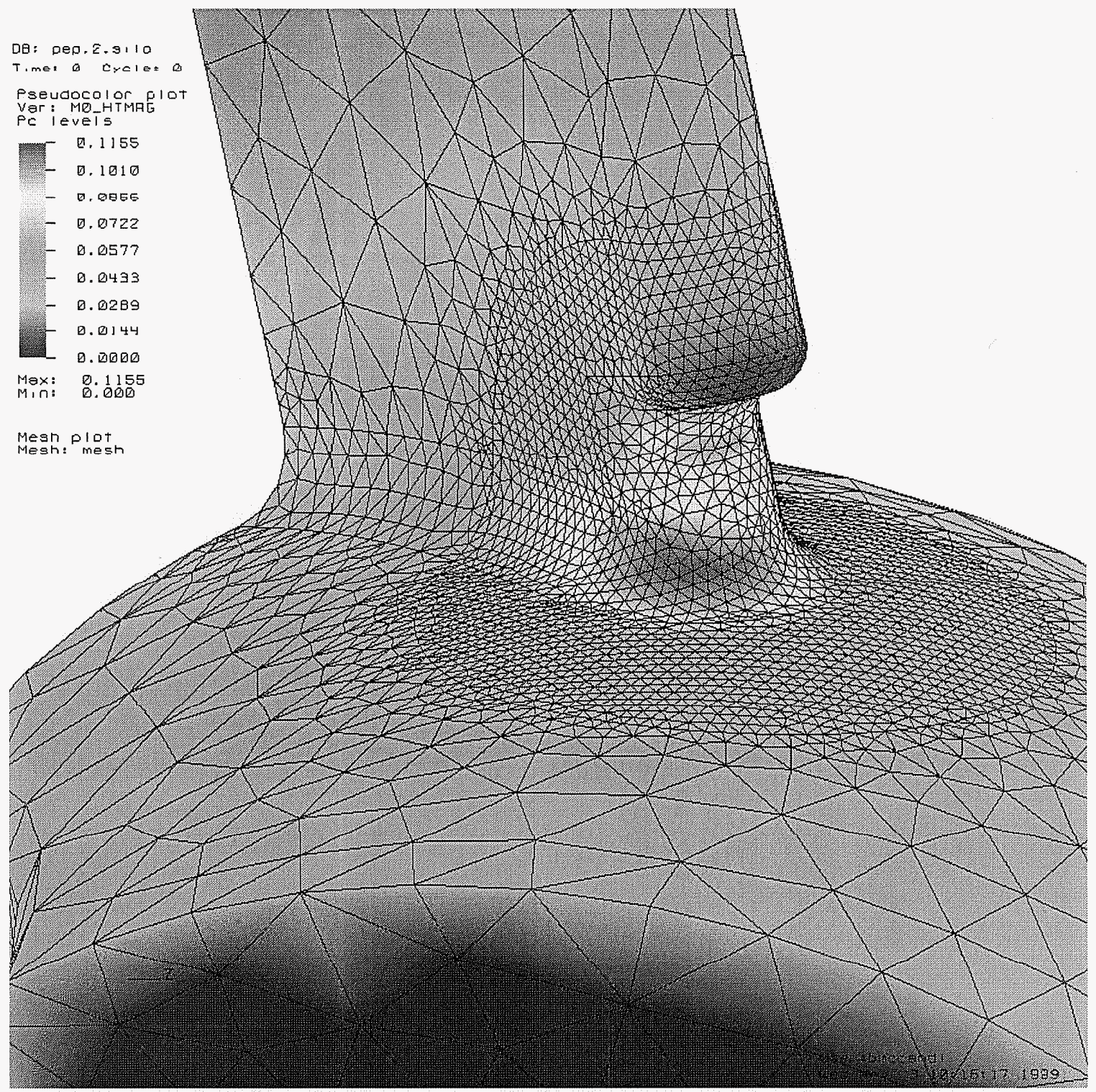

Figure 20. PEP-II model with $2.5 \mathrm{~mm}$ local element size around hot spot.

Table 5. PEP-II results with $2.5 \mathrm{~mm}$ local element size

\begin{tabular}{|l|l|}
\hline mesh size (hot spot): & $2.5 \mathrm{~mm}$ \\
\hline mesh size (nose): & $5 \mathrm{~mm}$ \\
\hline mesh size (everywhere else): & $18 \mathrm{~mm}$ \\
\hline \# elements: & 43555 \\
\hline \# degrees of freedom: & 262162 \\
\hline frequency: & 477123035 \\
\hline Peak power density* & $1.3909 \mathrm{MW} / \mathrm{m}^{\wedge} 2$ \\
\hline
\end{tabular}

* normalized to $150 \mathrm{~kW}$ total wall loss 


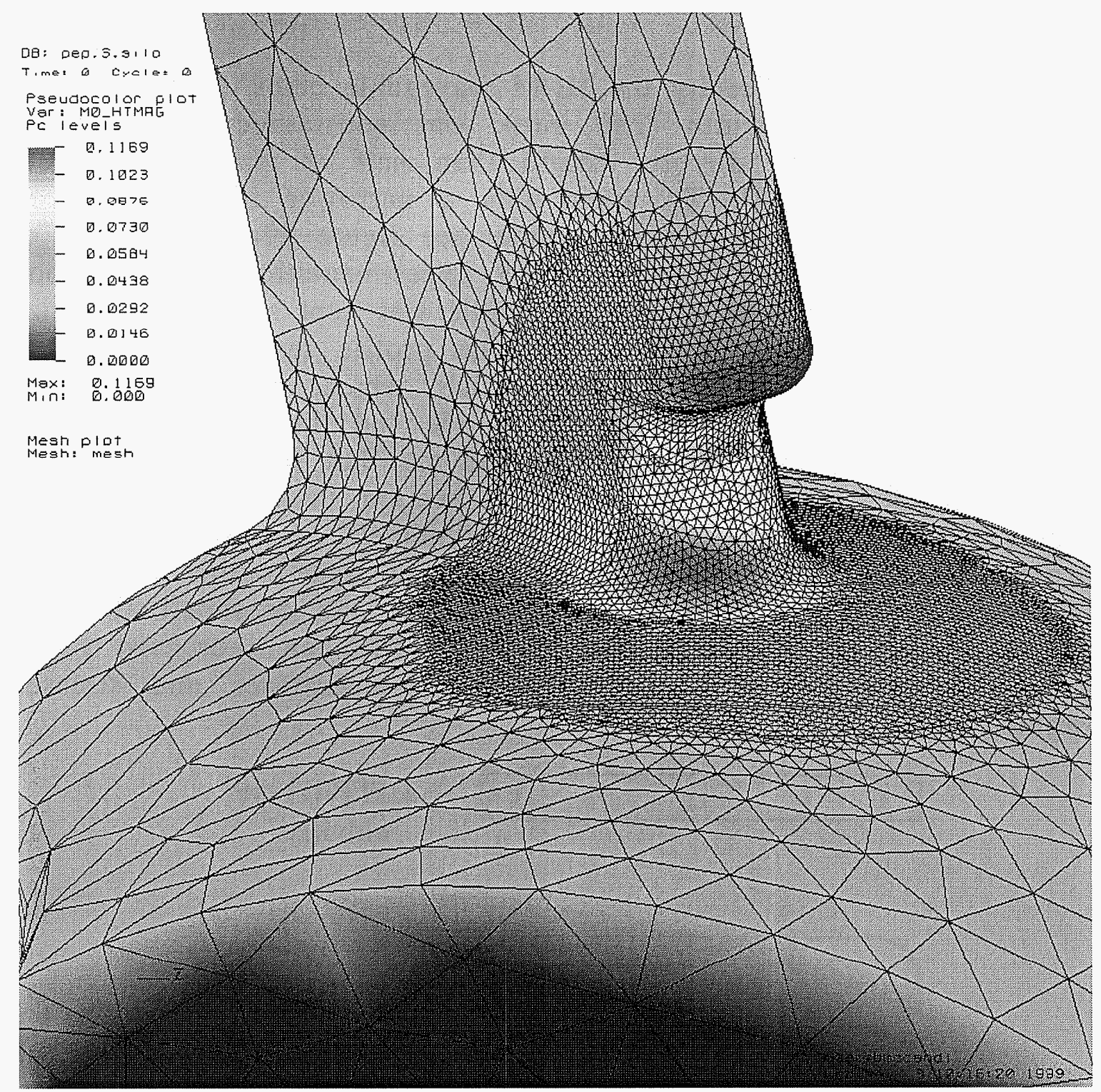

Figure 21. PEP-II model with $1.5 \mathrm{~mm}$ local element size.

Table 6. PEP-II results with $1.5 \mathrm{~mm}$ local element size

\begin{tabular}{|l|l|}
\hline mesh size (hot spot): & $1.5 \mathrm{~mm}$ \\
\hline mesh size (nose): & $5 \mathrm{~mm}$ \\
\hline mesh size (everywhere else): & $18 \mathrm{~mm}$ \\
\hline \# elements: & 106699 \\
\hline \# degrees of freedom: & 642758 \\
\hline frequency: & 477106196 \\
\hline Peak power density* & $1.3959 \mathrm{MW} / \mathrm{m}^{\wedge} 2$ \\
\hline
\end{tabular}

* normalized to $150 \mathrm{~kW}$ total wall loss 
Future changes

For the PEP-II cavity the HOM waveguide cutoff frequency was $600 \mathrm{MHz}$ and the width $(250 \mathrm{~mm})$, was considered too great to penetrate the cavity directly (more than half of the wall current would be intercepted). To avoid this a narrower iris was placed between the cavity and the waveguide, see figure 22a. The width of the coupling iris was determined by experimentation on a pillbox model [17]. The height of the waveguide was kept small to minimize loss of cavity wall area. The wide flat profile resulted in a small radius of curvature at the ends of the iris, which, combined with the small blend radius between the waveguide and the cavity body, led to a significant current concentration and the "hot spot" on the cavity wall. Possible variations on this cross section include a taller slot, figure $22 \mathrm{~b}$, or the use of a ridged "dumbbell" profile, figure $22 \mathrm{c}$, which results in a narrower guide for the same cutoff frequency. The increased height at the ends of the slot and dumbbell allows a greater radius of curvature and together with an increased blend radius reduces the peak surface losses, temperatures and stresses significantly.

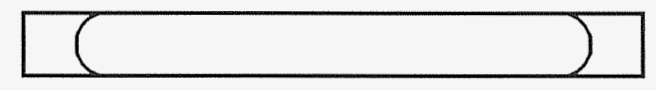
a. PEP-II type with iris

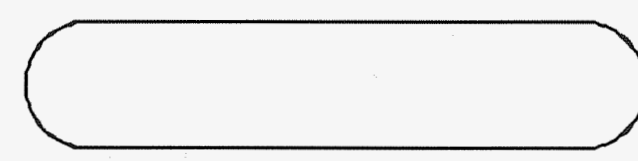

b. large slot

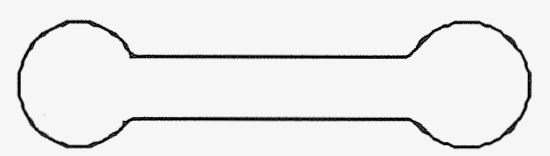

c. "dumbbell"

Figure 22. HOM waveguide cross sections with the same cut-off frequency $(600 \mathrm{MHz})$, a: PEP-II type rectangular guide (with iris), b: large slot, c: compact ridged "dumbbell" guide. 


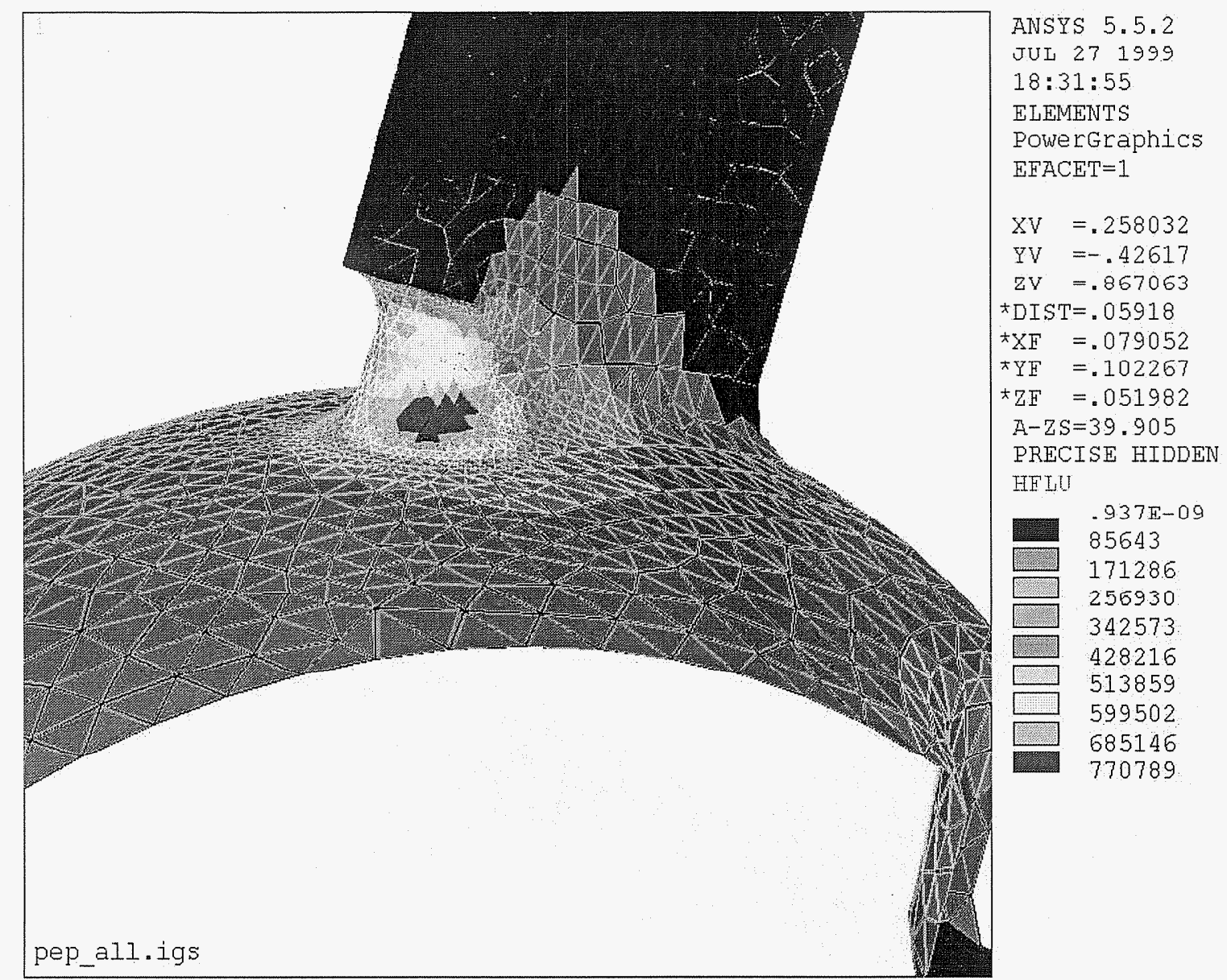

Figure 23. Heat flux for $714 \mathrm{MHz}$ PEP-type Iris Cavity Design.

As a reference design for work on a new $714 \mathrm{MHz}$ cavity for the NLC damping rings [6], a $2 / 3$ scale model of the PEP shape was run, resulting in a total cavity dissipation of $41 \mathrm{~kW}$, or $6.8 \mathrm{~kW}$ per sixth. In this size, the iris dimensions are $142 \mathrm{~mm}$ long by $16.93 \mathrm{~mm}$ tall, with an $8.5 \mathrm{~mm}$ blend radius into the cavity. The maximum heat flux shown in figure 23 is $77 \mathrm{~W} / \mathrm{cm}^{2}$, and the "hotspot" shows the characteristic shape with two maxima, previously observed for the $476 \mathrm{MHz}$ PEP-II cavity. Note that the contours are not completely smooth since this model was run with a comparatively large mesh size, and the heat flux values were not averaged.

Another HOM port shape investigated for the NLC cavity is the generic "slot" design, which consists of a simple, constant crosssection racetrack, with outside dimensions of $166.7 \mathrm{~mm}$ by 33.86 $\mathrm{mm}$. This racetrack shape is blended into the cavity with a 15.88 $\mathrm{mm}$ radius (5/8") all the way around. This port is much longer than the PEP iris, but it allows a much larger blend radius, and this radius turns out to be the dominant dimension in heat concentration. As can be seen in figure 24 , the maximum heat flux in the slot port is 
approximately $61 \mathrm{~W} / \mathrm{cm}^{2}$, which is more than $20 \%$ lower than the iris design.

The "dumbbell" HOM port design utilizes a wide blend radius, reduced width and a narrower middle section. The overall dimensions of the port in this analysis are $139.7 \mathrm{~mm}$ by $33.86 \mathrm{~mm}$, with the same blend radius as the slot design, $15.88 \mathrm{~mm}$. (Note that these dimensions were adjusted slightly for the final design). Studies showed that the slot achieves the best HOM damping but the dumbbell results are similar and both are better than the iris. The dumbbell succeeds in reducing the maximum heat flux to just under $52 \mathrm{~W} / \mathrm{cm}^{2}$, see figure 25 , a $15 \%$ improvement in heat dissipation compared to the slot and $33 \%$ lower than the iris, a significant advantage in the mechanical design.

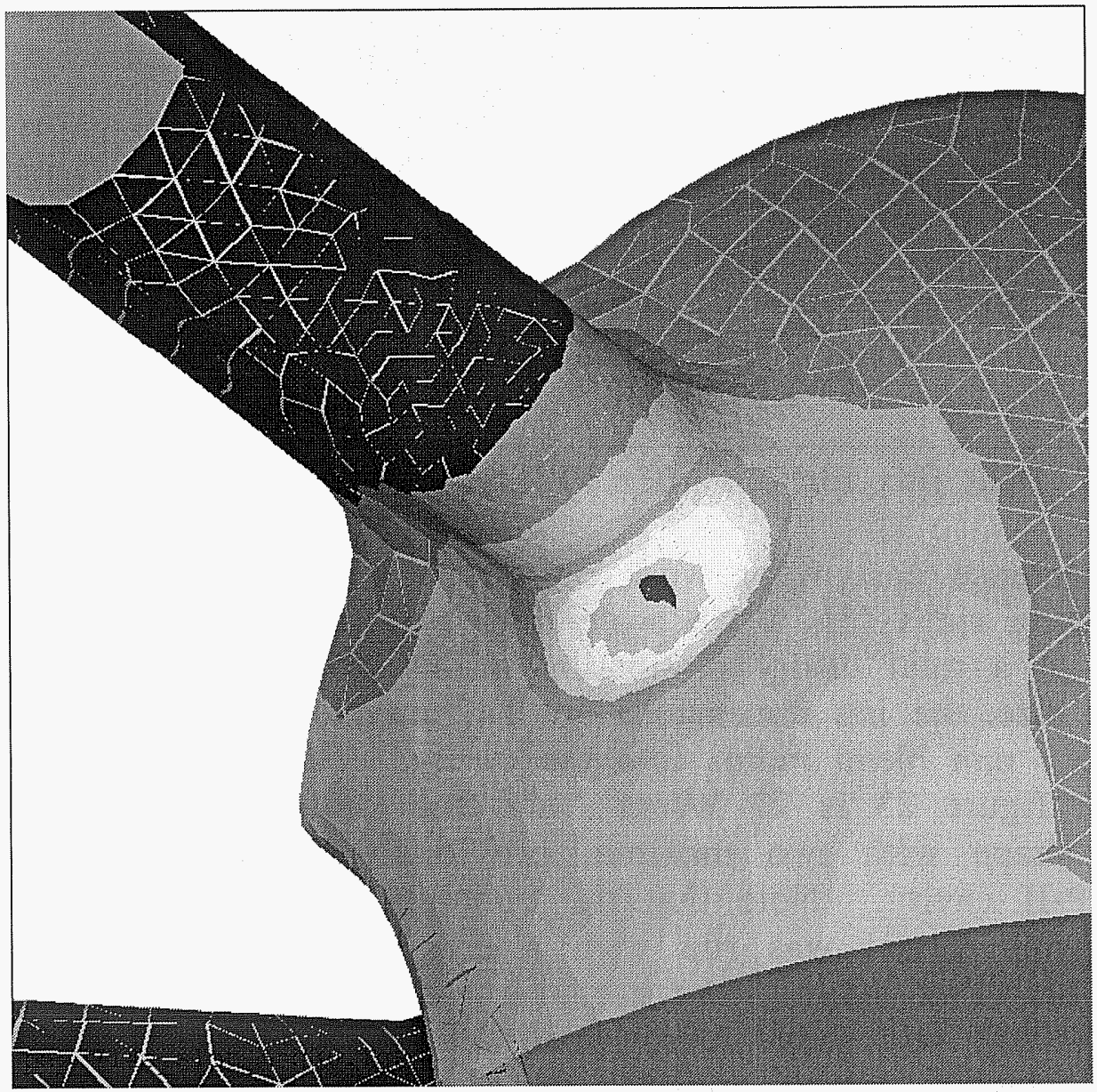

ANSYS 5.5 .2

JUL $30 \quad 1999$

15:45:07

ELEMENTS

PowerGraphics

EFACET $=1$

$X V=-.470194$

$\mathrm{YV}=.160025$

$\mathrm{zV}=.867934$

$\star$ DIST $=.073878$

$\star X F=-.089029$

¿YF $=.106671$

太ZF $=.040872$

$A-Z S=-8.582$

PRECISE HIDDEN

EDGE

HFLU

0
57334
13466

134668

269336

336671

404005

471339

538673

606007

Figure 24. Heat flux for alternative HOM "Slot" Design (714 MHz). 


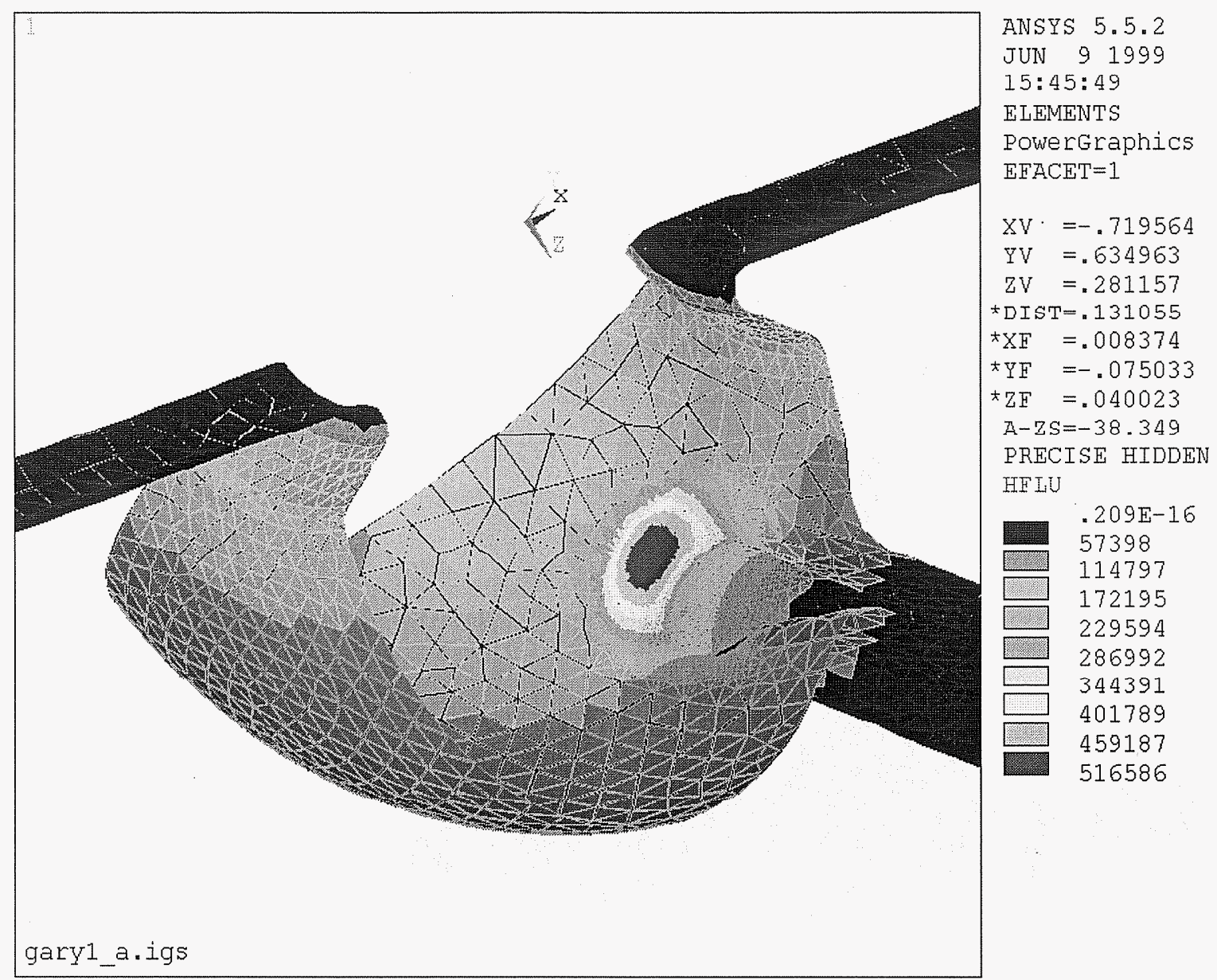

Figure 25. Heat flux for "dumbbell" HOM Port Design (714 MHz).

The improvements discussed above have been applied to the $714 \mathrm{MHz}$ NLC damping ring cavity. The HOM damping is as good or better than a scaled version of the PEP-II design, the temperatures and stresses are lower and the mechanical design is simpler. Figure 26 shows the wall power losses normalized to a total of $42 \mathrm{~kW}$, the expected dissipation for a gap voltage of $500 \mathrm{kV}$. (Note that the heat flux is slightly higher than in figure 25 because the width of the dumbbell was increased to improve the HOM damping). The maximum value at the hot spot is about $55 \mathrm{~W} / \mathrm{cm}^{2}$, significantly lower than the scaled PEP-II geometry, $77 \mathrm{~W} / \mathrm{cm}^{2}$. Figure 27 shows the temperature rise above the cooling channel temperature for this heat flux. Note the temperature at the HOM port hot spot is less than $30^{\circ} \mathrm{C}$ above the water temperature. (Compare with $64^{\circ} \mathrm{C}$ rise for the PEP-II cavity at it's maximum design value of $150 \mathrm{~kW}$ [18], $42^{\circ} \mathrm{C}$ at its normal operating power of $103 \mathrm{~kW}$ ). The water flow rate in the channels is assumed to be 4.1 GPM $(15.5 \mathrm{l} / \mathrm{min}$.) per channel with a Reynolds number of 34,000 and a heat transfer coefficient of 18000 
$\mathrm{W} / \mathrm{m}^{2}{ }^{\circ} \mathrm{C}$. The temperature rise in each channel is estimated to be less than $4^{\circ} \mathrm{C}$, but this small change was not included in the simulations.

Figure 28 shows the Von Mises stress in the body calculated by ANSYS. Note that the stress at the hot spot is about $35 \mathrm{MPa}$ (about $5000 \mathrm{Psi}$ ) which is well within the fatigue limit of copper for 10,000 cycles, which is about 18,000 Psi [19]. This stress can be compared with approximately 10,900 Psi $(75 \mathrm{MPa})$, estimated for the PEP-II cavities at $150 \mathrm{~kW}$. The model does not include atmospheric pressure but this is a small contribution to the total stress.

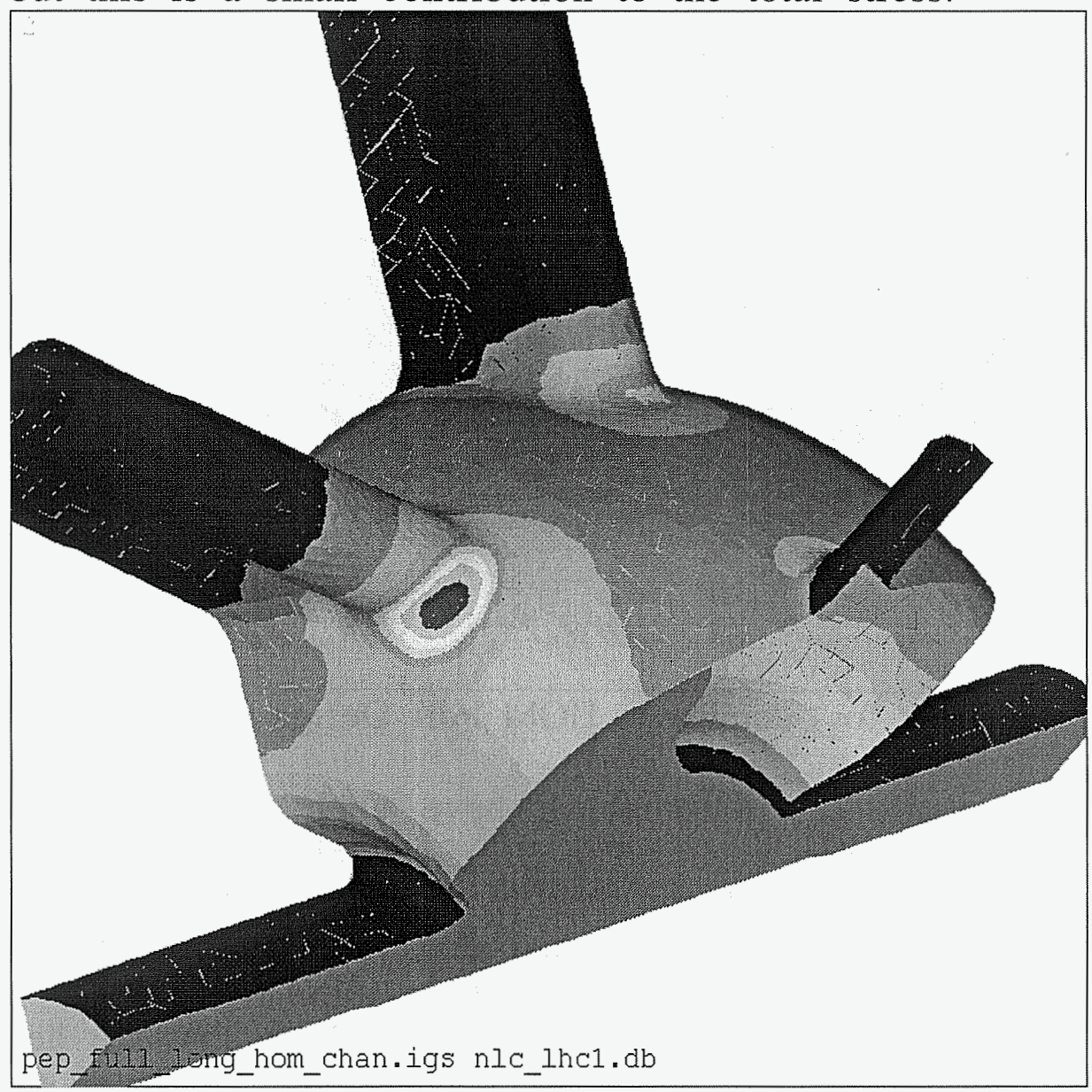

ANSYS 5.5 .2

NOV 41999

$10: 40: 49$

ELEMENTS

PowerGraphics

EFACET $=1$

WIND $=2$

$X V=-.651186$

$\mathrm{YV}=-.070283$

$Z V=.755657$

DIST $=.150225$

$\star \mathrm{XF}=-.08245$

$\star Y F=-.114872$

$+\mathrm{ZF}=-224.72$

$A-Z S=-166.243$

PRECISE HIDDEN

HFF LU

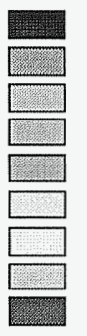

0

61016

122031

183047

244062

305078

366093

427109

488124

549140

Figure 26. Surface power loss on proposed NLC cavity. 


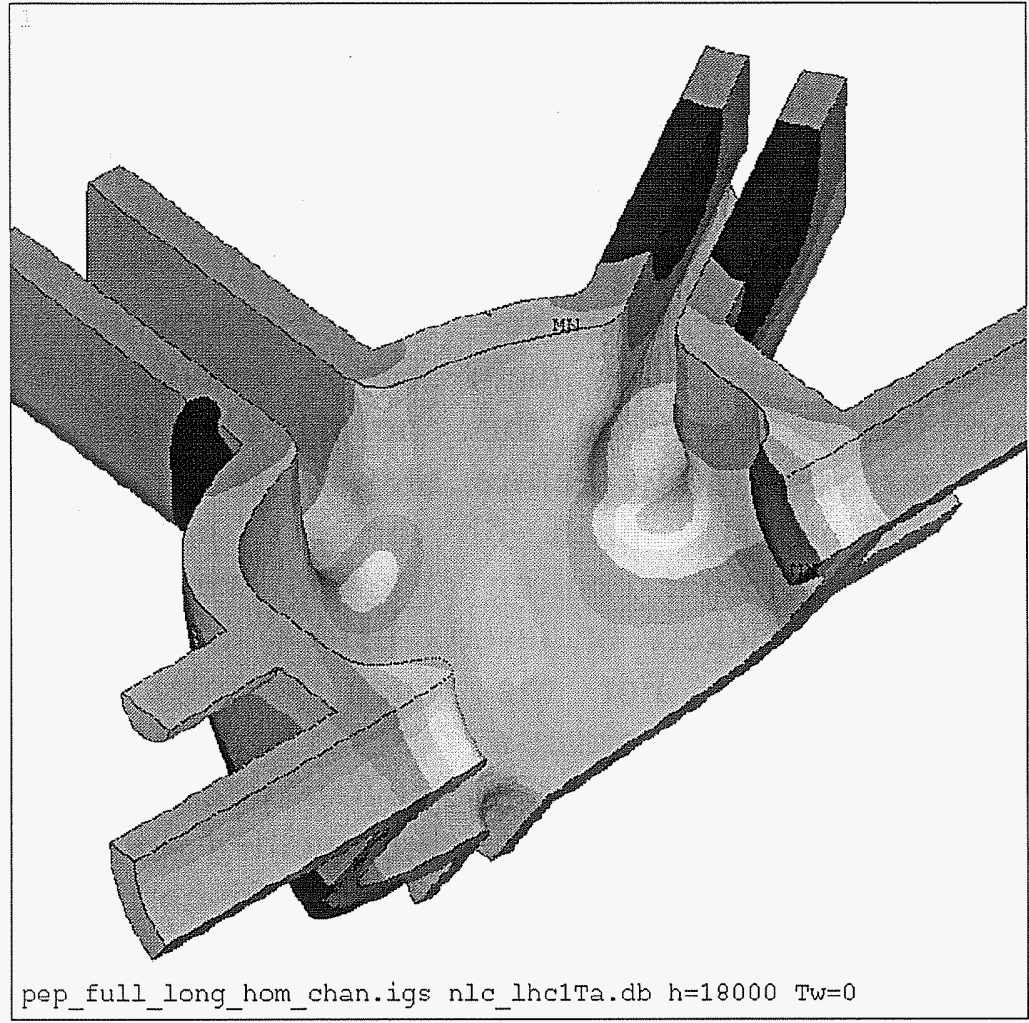

ANSYS 5.5 .2

NOV 51999

$14: 16: 11$

NODAL SOLUTION

$S T E F=1$

SUB $=1$

TIME $=1$

TEMP

(AVG)

RSYS $=0$

Powergraphics

EFACET $=1$

AVRES=Mat

SMN $=0$

$\operatorname{SMX}=32.144$

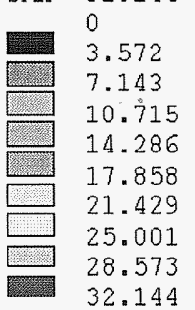

Figure 27. Surface temperature of the NLC cavity calculated by ANSYS.

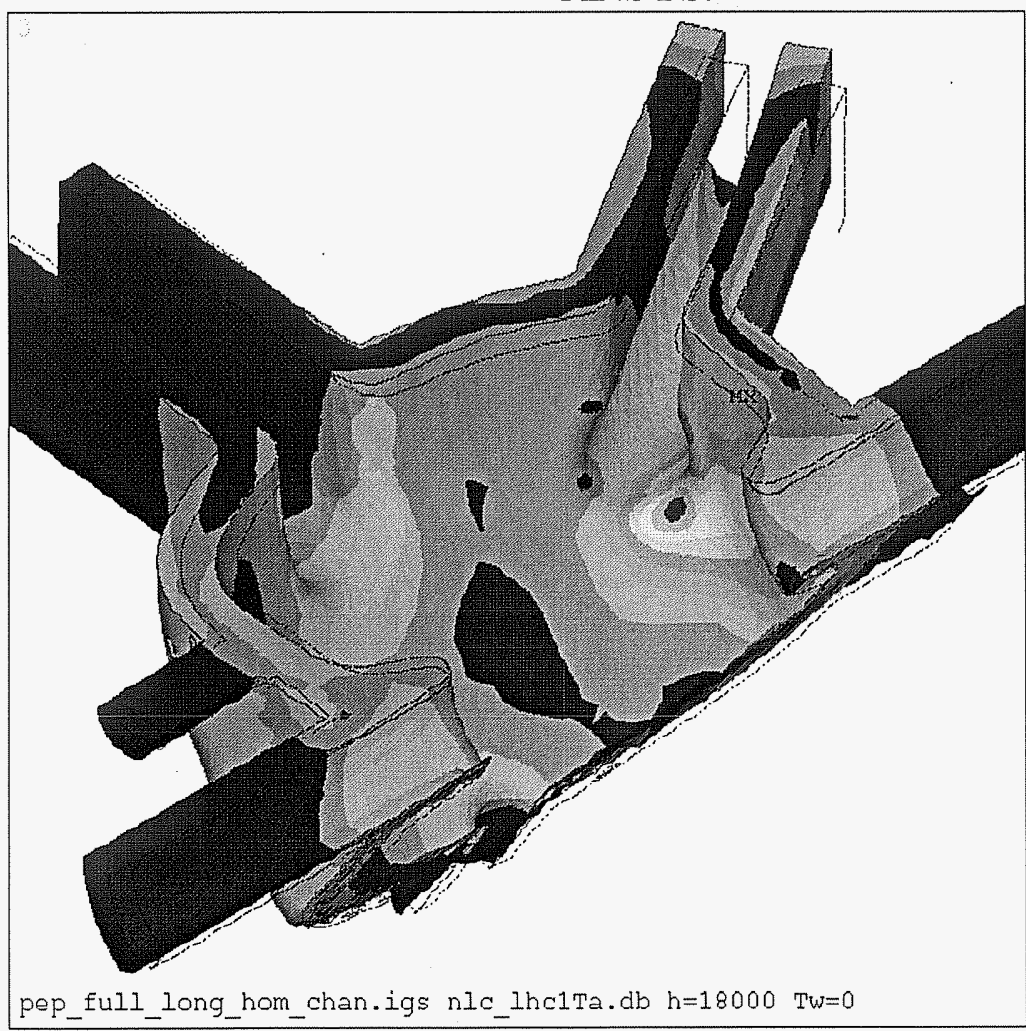

ANSYS 5.5 .2

NOV 51999

$17: 28: 38$

NODAL SOLUTION

STEF $=1$

SUB $=1$

TIME $=1$

SEQV (AVG)

$\mathrm{DMX}=.774 \mathrm{E}-04$

SMN $=320.225$

$\operatorname{SMX}=.754 \mathrm{E}+08$

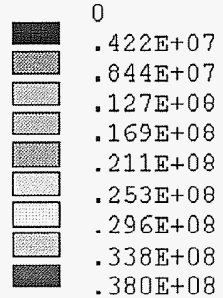

Figure 28. Von Mises stress in the NLC cavity body calculated by ANSYS. 


\section{Fabrication}

The PEP-II cavity mechanical design evolved over several years from the original concept of an all-brazed assembly to one utilizing many different fabrication and joining methods, including brazing, e-beam welding, TIG welding, TIG brazing and electroplating. Some of these processes were applied to multiple separate parts; for example, the body main section and the lid section were both electroplated to complete the cooling channels but this was done on each part separately before they were joined together by e-beam welding. Similarly, each of the six ports and both nosecones had their own internal cooling channels which were made by brazing on close fitting jackets or inserts before the ports were ebeam welded into the body. For the much simpler ALS third harmonic cavities, figure 29 , this process was revised. The equatorial ports were made integral to the cavity body, facilitated by the small size and spherical profile, the end caps were welded into the body before plating and all cooling channels were finished and water fittings grown in with one plating operation. (There were no HOM ports but the same process might be applied if there were).

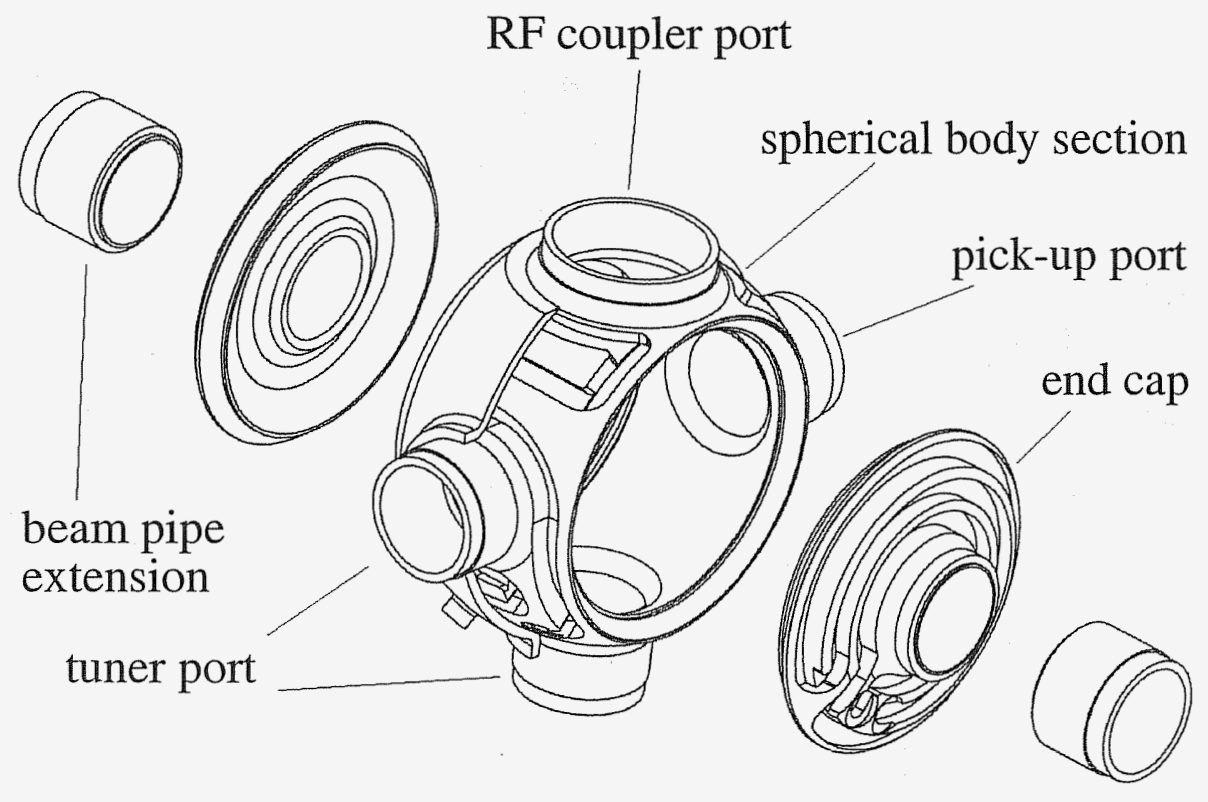

Figure 29 ALS third harmonic cavity assembly.

The electroplating process for the ALS cavities required some development to get the placement of electrodes correct for uniform plating coverage, but if more than a few cavities are produced then 
this cost is more than offset by the savings from the eliminated parts and operations.

The proposed construction process for the NLC RF cavities is based on the fabrication of 2 major components, the cavity lid and body, as shown in figure 30. They are made from C10100 alloy copper forgings sized to incorporate the finished surface geometry of each component. When developed by a series of machining processes, both represent monolithic structures with completely developed internal and external features. The two components are then joined together by electron beam welding to form a single unit, and are then ready to accept the port flanging by the same weld process. The forged and cold-worked assembly presents a robust copper structure without any reduction in strength from brazing or annealing.

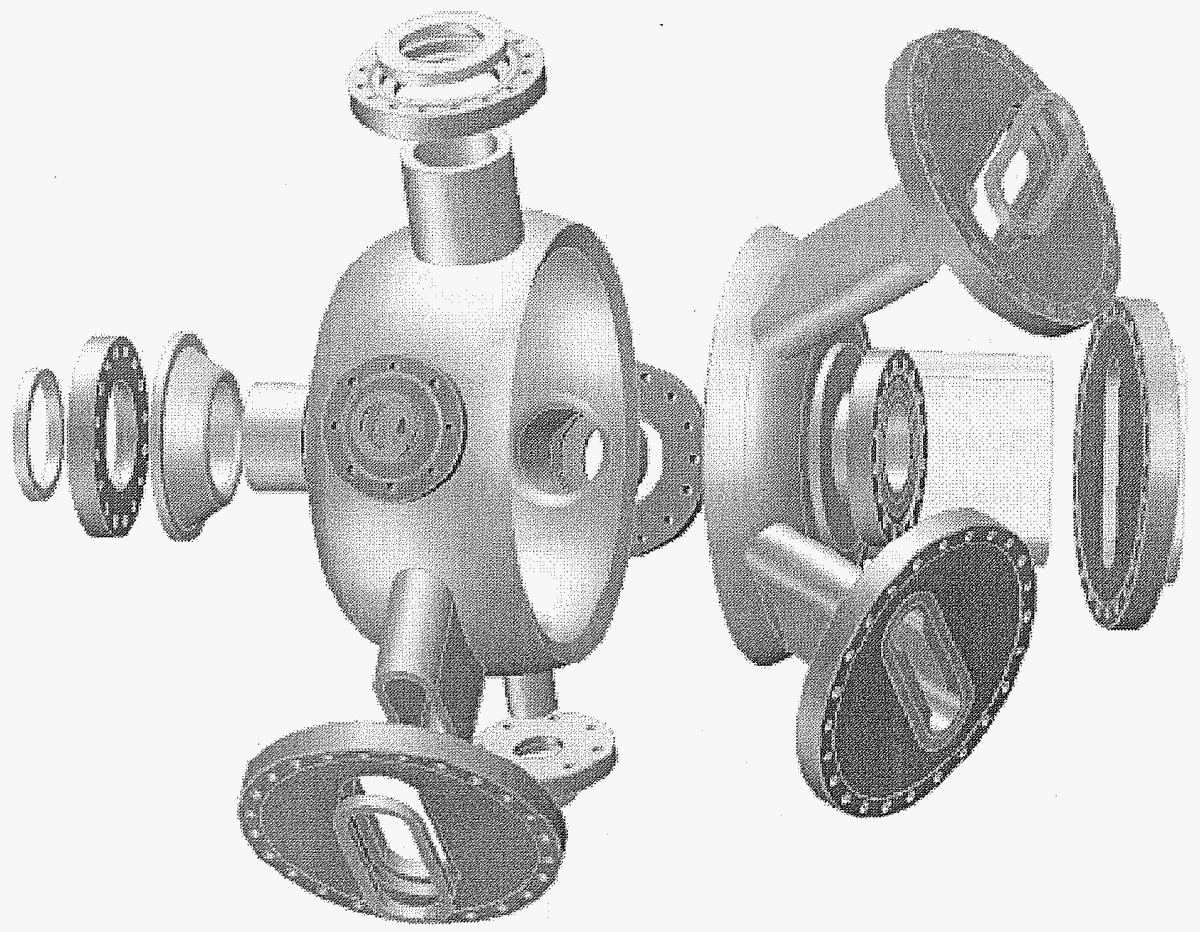

Figure 30. Assembly of proposed NLC cavity.

This construction technique is a departure from the PEP-II fabrication approach. By reducing the number of subassemblies and weld attachments, the assembly costs should be greatly reduced in the NLC design. As a point of reference, the number of weld and hydrogen braze processes are reduced from approximately 42 major elements on PEP-II to 24 on NLC design. Of these 24, 11 are supplied by a commercial vendor as part of the port flanging procurement. 
Conclusions

The PEP-II cavity is a very successful design that is operating as expected and has made an important contribution to the rapid commissioning of the B factory. The HOM performance predicted by initial calculations and laboratory measurements has been verified by observations of the beam induced signals and the operation of the feedback systems, which record HOM driven instability growth rates and thresholds in the ranges expected. Better computational tools have been applied to the design and show good agreement with the known performance, giving confidence in the application of those methods to future designs. The calculation of the surface heating using ANSYS shows good agreement with the original Chalk River results and with those from the state of the art Omega3p code at SLAC. The analyses show the importance of good mesh refinement at the hot spots. The wide availability of ANSYS and the rapid turnaround of results on even modest PC based workstations make this a very useful tool for future projects. The ANSYS analysis highlighted a discrepancy between the original numerical model and the as-built design resulting in an approximately $10 \%$ higher stress in reality than previously thought. The PEP-II cavities are presently being operated well within their limitations, however, and this is not a cause for concern. The stress analyses also shows the importance of the physical constraints at the ports, which must be free to move in response to thermal expansion of the cavity, otherwise the stresses in the body may be as much as doubled.

It is clear that simple modifications of the cavity geometry, particularly in the region of the HOM ports could significantly lower the peak RF losses, temperatures and stresses. For future applications the successful waveguide damping scheme may be adapted by using larger radii at the ends of the slots and for the blend into the cavity, thereby maintaining or improving the HOM damping while substantially reducing the peak temperatures and stresses in the body. The "dumbbell" shaped ridged waveguide is a good compromise with a reasonably small width and reasonably large radius at the ends.

The fabrication processes used to build the PEP-II cavities can be adapted and refined to make future cavities simpler and cheaper without sacrificing performance. 
References

[1] "Commissioning Results Of The KEKB And PEP-II B-Factories", J.T. Seeman, Proceedings of the 1999 Particle Accelerator Conference, New York, 1999.

[2] "DAфNE Operating Experience", S. Guiducci, Proceedings of the 1999. Particle Accelerator Conference, New York, 1999.

[3] "A Third-Harmonic RF Cavity for the Advanced Light Source", R.A. Rimmer, EPAC98, Stockholm.

[4] "Design Considerations for a Second Generation HOM-Damped RF Cavity", R. A. Rimmer Proceedings of the 1999 Particle Accelerator Conference, New York, 1999.

[5] "Zeroth-Order Design Report for the Next Linear Collider", LBNL-PUB-5424, SLAC report 474, UCRL-ID-124161.

[6] "RF cavity R\&D at LBNL for the NLC Damping Rings, FY1999", R.A. Rimmer et. al., CBP Tech Note 196, Nov. 1999.

[7] "Computer Determination of the External $Q$ and Resonant Frequency of Waveguide Loaded Cavities", N. Kroll, D. Yu, Part.Accel.34:231-250,1990, SLAC-PUB-5171, January 1990.

[8] "Updated Impedance Estimate of the PEP-II RF Cavity", R.A. Rimmer et. al., Proc EPAC 96, Spain.

[9] "Impedance Spectrum for the PEP-II RF Cavity", X.E. Lin, K. Ko, C.K. Ng, Proc. PAC 95, Dallas, TX, SLAC-PUB-95-6902, PEP-II AP-Note-95.31, 21-JUN-95.

[10] "Commissioning Experience from PEP-II HER Longitudinal Feedback", S. Prabhakar, D. Teytelman, J. Fox, A. Young, P. Corredoura, R. Tighe. SLAC-PUB-7849, Jun 1998. 9pp. Poster paper at 8th Beam Instrumentation Workshop (BIW 98), Stanford, CA, 4-7 May 1998.

[11] "Notes from the RF cavity production close-out meeting, LLNL, 11/13/97", R.A. Rimmer et. al., PEP-II EE Note 97.07. 
[12] "Cavity RF Mode Analysis Using a Boundary-Integral Method", M.S. De Jong, F.P. Adams, Proc, 1993 PAC, Washington DC, pp $835-7$.

[13] Ansys, Inc., 275 Technology Drive, Canonsburg, PA 15317 Tel: 724-746-3304, Fax: 724-514-9494 (http://www.ansys.com).

[14] HF 119, High Frequency Electromagnetic Tetrahedra, ANSYS Elements Manual Section 10.2.

[15] "Omega3p: A Parallel Eigensolver of Modeling Large, Complex Cavities", Proc. ICAP 98, Monterey, CA.

[16] John Hodgson, SLAC Memorandum, May 18, 1994.

[17] "Higher Order Mode Damping in a Pillbox Cavity", F. Voelker et. al., Proc. 1991 PAC, San Francisco, pp 687-9.

[18] "High-Power RF Cavity R\&D for the PEP-II B-Factory", R. Rimmer et. al., Proc. EPAC 94, London.

[19] "Low temperature mechanical properties of copper and selected copper alloys; a compilation from the literature", Richard P. Reed and Ritchie P. Mikesell, [Washington] U.S. Dept. of Commerce, National Bureau of Standards; for sale by the Supt. of Docs., U.S. Govt. Print. Off., 1967, p43. 